\title{
Mixed convection in a Falkner-Skan system
}

\author{
J. H. Merkin
}

Received: 25 July 2015 / Accepted: 13 December 2015 / Published online: 14 March 2016

(C) The Author(s) 2016. This article is published with open access at Springerlink.com

\begin{abstract}
The effects of mixed convection on the classical Falkner-Skan similarity solutions are considered, now involving a mixed convection parameter $\lambda$ as well as the exponent $m$ associated with the outer flow. The forced convection solutions indicate a singularity in the temperature field as $m \rightarrow 0.070722$. Numerical solutions for $m>0$ show the existence of a critical value $\lambda_{\mathrm{c}}$ with $\lambda_{\mathrm{c}}<0$ and solutions only for $\lambda \geq \lambda_{\mathrm{c}}$. The nature of the solution for $\lambda \gg 1$ is investigated. For $m=0$, there are solutions for all $\lambda<0$, opposing flow, and only for a finite range of $\lambda$ in aiding flow with the asymptotic solution as $\lambda \rightarrow-\infty$ also being considered. Solutions for $m<0$ are obtained in the cases when there is a solution to the Falkner-Skan system and for a value of $m$ when no solution to this system exists. In the former case, two completely separate parts to the solution are seen, whereas in the latter case, a solution exists only in aiding flow for a limited range of $\lambda$. The variation of solution with the exponent $m$ is also treated for both aiding and opposing flows. In both cases, a solution is seen to exist for all $m>0$, which, however, is limited to a relatively small range of $m$ when $m<0$.
\end{abstract}

Keywords Boundary-layer flow $\cdot$ Falkner-Skan system $\cdot$ Mixed convection

Mathematics Subject Classification 76D10

\section{Introduction}

The original work by Falkner and Skan [1] presented a classical boundary-layer similarity solution, their results being extended by Hartree [2], see also Rosenhead [3]. In this, a free stream $U_{\infty}(x) \propto x^{m}$ flows over a fixed impermeable surface, where $x$ measures distance along the bounding surface. This form for the outer flow allows the problem to be reduced to a similarity system, more usually characterized by the parameter $\beta=2 m /(m+1)$. The problem was later considered in more detail by Stewartson [4] who showed that there was a lower bound $\beta_{\mathrm{c}}$ of $\beta$ for the existence of a solution, with $\beta_{\mathrm{c}} \simeq-0.19884$, and dual solutions in $\beta_{\mathrm{c}}<\beta<0$. The singular nature of the lower branch solutions as $\beta \rightarrow 0$ from below was derived by Brown and Stewartson [5]. Further solutions to this basic problem have been obtained by Craven and Peletier [6] for $\beta>1$ (in the present notation) some exhibiting several regions of reversed flow. There can also be branching solutions when $\beta<\beta_{\mathrm{c}}$ as exhibited by Oskham

J. H. Merkin $(\bowtie)$

Department of Applied Mathematics, University of Leeds, Leeds LS2 9JT, UK

e-mail: amtjhm@maths.leeds.ac.uk 
and Veldman [7], building on the previous work of Libby and Lui [8], with the possibility of periodic solutions for $\beta<-1$ being reported. More recently Fang et al. [9] have derived an algebraic solution to the Falkner-Skan problem for the particular case $m=-\frac{1}{3}$, or $\beta=-1$.

An alternative formulation of the problem is to allow the bounding surface to be moving in a direction along its length with a velocity $U_{\mathrm{s}}(x) \propto x^{m}$ to preserve the similarity form though now without an outer flow. This situation was originally discussed by Crane [10] and Banks [11] and has subsequently been extended in many different ways, for example, to include wall transpiration [12,13], wall slip velocities [14] and the effects of magnetic fields [15]. Many of these solutions as well as some further exact solutions are presented in the recent papers by Wang [16], Al-Housseiny and Stone [17] and Magyari [18]. The situation when there are in effect both a moving wall and an outer flow of the forms described above has arisen in several different contexts, particularly the case when $m=0$ (or $\beta=0$ ) and has been much discussed, see, for example, Klemp and Acrivos [19], Merrill et al. [20], Hussaini et al. [21] and Riley and Weidman [22]. Here there is a further parameter $\epsilon$ (say) which gives the ratio of the wall velocity to that of the outer flow. Critical values $\epsilon_{\mathrm{c}}$ of $\epsilon$ are found, dependent on $m$, which limit the solution to $\epsilon \geq \epsilon_{\mathrm{c}}$ with the saddle-node bifurcation at $\epsilon=\epsilon_{\mathrm{c}}$ giving a range of $\epsilon$ where there are dual solutions.

Mixed convection arises when there is an interaction between the flow and a temperature field within the boundary layer. This temperature variation, which usually arises from some applied temperature or heat flux distribution on the boundary, sets up buoyancy forces which can either aid or oppose the development of the boundary-layer flow and can have significant effects on both the flow and heat transfer, see [23-26], for example. Here we consider a modification to the Falkner-Skan problem to include the mixed convection arising from the application of a prescribed temperature distribution on the surface. If we choose a surface temperature $T_{\mathrm{w}} \propto x^{2 m-1}$, then the similarity nature of the problem is maintained. This introduces a further (dimensionless) mixed convection parameter $\lambda$, defined below, into the equation for the flow with the solution now depending on both $m$ and $\lambda$. Here we keep the exponent $m$ directly in our equations, rather than using $\beta$ mentioned above, so as to give a more direct relation between the outer flow and the functional form of the prescribed wall temperature. Our aim is to examine how the solution to this modified Falkner-Skan system behaves over the $m-\lambda$ parameter plane, paying particular attention to those ranges of the mixed convection parameter $\lambda$ where a solution can exist, finding that these depend to a large extent on the exponent $m$. We start by deriving the similarity equations.

\section{Equations}

The Falkner-Skan similarity solutions for the boundary-layer equations are based on an outer flow of the form $U(x) \propto x^{m}$ for some exponent $m$, where $x$ measures distance along the bounding surface $[1,3,4]$. Consequent on this form of outer flow, to obtain a similarity solution for mixed convection on a planar vertical surface, we require a prescribed wall temperature $T_{\mathrm{w}}$ of the form $T_{\mathrm{w}}-T_{\infty} \propto x^{2 m-1}$, where $T_{\infty}$ is the (constant) ambient temperature. This leads to the boundary conditions:

$\psi=\frac{\partial \psi}{\partial y}=0, \quad T-T_{\infty}=T_{0}\left(\frac{x}{\ell}\right)^{2 m-1} \quad$ on $y=0, \quad \frac{\partial \psi}{\partial y} \rightarrow U_{0}\left(\frac{x}{\ell}\right)^{m}, \quad T \rightarrow T_{\infty} \quad$ as $y \rightarrow \infty$,

where $\psi$ is the streamfunction defined in the usual way, and $y$ measures the distance normal to the surface. In (1), $\ell$ is a length scale, $U_{0}>0$ is a velocity scale and $T_{0}$ a temperature scale which can be either positive (aiding flow) or negative (opposing flow).

To make the steady, two-dimensional boundary-layer equations for mixed convection flow on a vertical surface, see [27], for example, subject to boundary conditions (1) dimensionless, we introduce the variables

$\psi=\ell \operatorname{Re}^{-1 / 2} U_{0} \bar{\psi}, \quad T-T_{\infty}=T_{0} \bar{T}, \quad \bar{x}=\frac{x}{\ell}, \quad \bar{y}=\operatorname{Re}^{1 / 2} \frac{y}{\ell}$,

where $\operatorname{Re}=\left(U_{0} \ell\right) / v$ with $v$ is the kinematic viscosity. Then, to reduce the problem to similarity form, we make the further transformation, on dropping the overbars,

$\psi=x^{(m+1) / 2} f, \quad T=x^{2 m-1} \theta, \quad \eta=y x^{(m-1) / 2}$. 
This gives the similarity system

$$
\begin{aligned}
& f^{\prime \prime \prime}+\frac{(m+1)}{2} f f^{\prime \prime}+m\left(1-f^{\prime 2}\right)+\lambda \theta=0, \\
& \frac{1}{\sigma} \theta^{\prime \prime}+\frac{(m+1)}{2} f \theta^{\prime}-(2 m-1) f^{\prime} \theta=0,
\end{aligned}
$$

subject to

$f(0)=f^{\prime}(0)=0, \quad \theta(0)=1, \quad f^{\prime} \rightarrow 1, \quad \theta \rightarrow 0 \quad$ as $\eta \rightarrow \infty$,

where primes denote differentiation with respect to $\eta$ and where the mixed convection parameter $\lambda=\left(g \beta \ell T_{0}\right) / U_{0}^{2}$. In Eq. (5), $\sigma$ is the Prandtl number and, for simplicity, we take $\sigma=1$ throughout. At this stage, we put no restriction on the exponent $m$.

\section{Results}

\subsection{Forced convection, $\lambda=0$, limit}

We start by considering the solution to Eqs. (4-6) for the case when $\lambda=0$. Then Eq. (4) is the standard FalknerSkan problem, usually written in terms of $\beta=2 m /(m+1)$ rather than $m$, the exponent of the outer flow [3]. In Fig. 1a (for completeness), we plot the values of $f^{\prime \prime}(0)$ against $m$ obtained from the numerical solution of $(4,6)$. The graph indicates a saddle-node bifurcation at $m=m_{\mathrm{c}} \simeq-0.09043$, corresponding the value $\beta \simeq-0.19884$ given previously [4], leading to two solution branches in $m_{\mathrm{c}}<m<0$. We note that, at the saddle-node bifurcation, $f^{\prime \prime}(0)=0$, changing from positive to negative. The upper branch solution can be continued to become large $m$ (noting that we obtained numerical results for much larger values of $m$ than are used to plot Fig. 1a). To determine the nature of the solution for $m$ large, we put $f=m^{-1 / 2} \bar{f}, \bar{\eta}=m^{1 / 2} \eta$. The leading-order problem is then

$\bar{f}^{\prime \prime \prime}+\frac{1}{2} \bar{f} \bar{f}^{\prime \prime}+1-\bar{f}^{2}=0, \quad \bar{f}(0)=\bar{f}^{\prime}(0)=0, \bar{f}^{\prime} \rightarrow 1 \quad$ as $\bar{\eta} \rightarrow \infty$,

where primes now denote differentiation with respect to $\bar{\eta}$. Eq. (7) has the solution $\bar{f}^{\prime \prime}(0)=1.19304$, giving $f^{\prime \prime}(0) \sim 1.19304 m^{1 / 2}+\cdots$ as $m \rightarrow \infty$. The lower branch solutions terminate in a singularity as $m \rightarrow 0$ from below [5].

We now consider Eq. (5) for the temperature $\theta$. We start by noting that, when $m=\frac{1}{5}$, Eq. (5) can be integrated to, on satisfying the outer boundary conditions,

$\theta^{\prime}+\frac{3}{5} \sigma f \theta=0$,

so that, in this case, $\theta^{\prime}(0)=0$, a result that also applies in the general, $\lambda \neq 0$, case. In Fig. 1b, we plot $\theta^{\prime}(0)$ against $m$. The occurrence of dual solutions arising from the saddle-node bifurcation at $m=m_{\mathrm{c}}$ is not clear in the figure but is seen in our numerical solutions, noting that $\theta^{\prime}(0)=0$ at this bifurcation. For $m$ large, we again put $\bar{\eta}=m^{1 / 2} \eta$ with the leading-order problem becoming

$\frac{1}{\sigma} \theta^{\prime \prime}+\frac{1}{2} \bar{f} \theta^{\prime}-2 \bar{f}^{\prime} \theta=0, \quad \theta(0)=1, \quad \theta \rightarrow 0 \quad$ as $\bar{\eta} \rightarrow \infty$.

Equation (9) has the solution $\theta^{\prime}(\bar{\eta}=0)=-0.91925$, giving $\theta^{\prime}(0) \sim-0.91925 m^{1 / 2}+\cdots$ as $m \rightarrow \infty$. These asymptotic results for large $m$ are shown in Fig. 1 by broken lines and appear to give good agreement with the numerical values even at quite moderate values of $m$.

However, the most significant feature of these results is that the solution becomes singular at $m=m_{0}$ with our numerical results suggesting that $m_{0} \simeq 0.07$ (indicated in Fig. 1 b by a broken line), with $\left|\theta^{\prime}(0)\right|$ becoming infinite, even though $f^{\prime \prime}(0)$ remains finite and passes smoothly through $m_{0}$. To describe how the solution behaves as $m \rightarrow m_{0}$, we put $m=m_{0}+\epsilon, \theta=\epsilon^{-1} h$ and then look a solution valid for small $\epsilon$ by expanding

$f(\eta ; \epsilon)=f_{0}(\eta)+\epsilon f_{1}(\eta)+\cdots, \quad h(\eta ; \epsilon)=h_{0}(\eta)+\epsilon h_{1}(\eta)+\cdots$. 

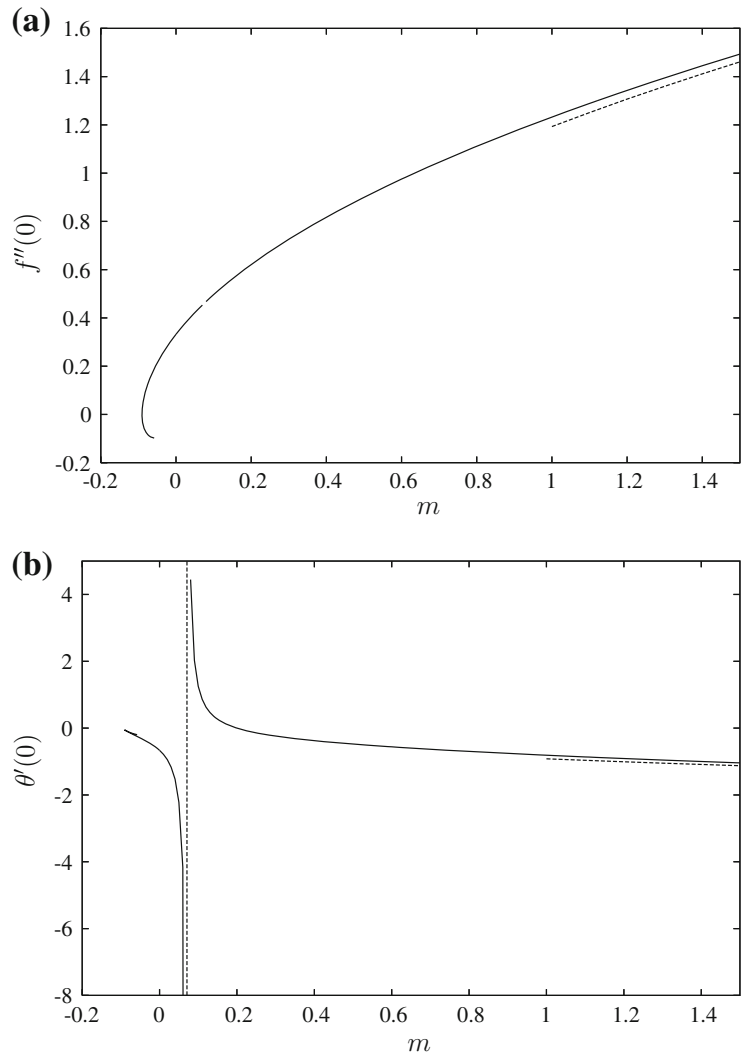

Fig. 1 Forced convection: plots of $\mathbf{a} f^{\prime \prime}(0)$ and $\mathbf{b} \theta^{\prime}(0)$ against $m$ for $\lambda=0$ and $\sigma=1.0$ obtained from the numerical solution of Eqs. (4-6) with the singularity in $\theta$ at $m=m_{0} \simeq 0.070722$ being indicated by a broken line. The asymptotic results for $m$ large are also shown by broken lines
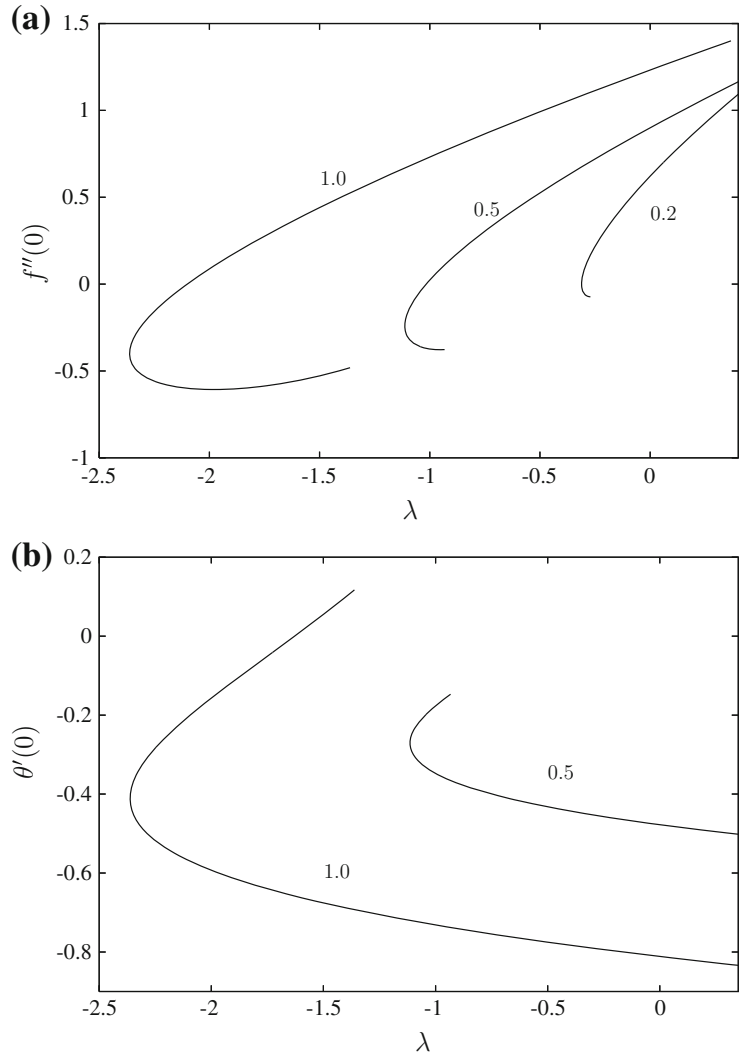

Fig. 2 Plots of a $f^{\prime \prime}(0)$ and $\mathbf{b} \theta^{\prime}(0)$ against $\lambda$ for $m=$ $1.0,0.5,0.2$ and $\sigma=1.0$ obtained from the numerical solution of Eqs. (4-6)

The leading-order problem is

$f_{0}^{\prime \prime \prime}+\frac{\left(m_{0}+1\right)}{2} f_{0} f_{0}^{\prime \prime}+m_{0}\left(1-f_{0}^{\prime 2}\right)=0$,
$\frac{1}{\sigma} h_{0}^{\prime \prime}+\frac{\left(m_{0}+1\right)}{2} f_{0} h_{0}^{\prime}-\left(2 m_{0}-1\right) f_{0}^{\prime} h_{0}=0$,

subject to

$f_{0}(0)=0, \quad f_{0}^{\prime}(0)=0, \quad h_{0}(0)=0, \quad f_{0}^{\prime} \rightarrow 1, h_{0} \rightarrow 0 \quad$ as $\eta \rightarrow \infty$,

where primes again denote differentiation with respect to $\eta$. Equation (11) is simply the Falkner-Skan problem for $m_{0}$ and this, together with Eq. (12) and the homogeneous boundary conditions on $h_{0}$, is an eigenvalue problem for $m_{0}$. To fix this, we add in the additional constraint that $h_{0}^{\prime}(0)=-1$ to obtain the specific solution $\bar{h}_{0}$. We solved this problem using the program D02AGF in the NAG library [28]. This gave $m_{0}=0.070722$ and $f_{0}^{\prime \prime}(0)=0.454072$.

In general, $h_{0}^{\prime}(0)=-a_{0}$ for some constant $a_{0} \neq 0$ so that $h_{0}=a_{0} \bar{h}_{0}$.

At $O(\epsilon)$, we have

$f_{1}^{\prime \prime \prime}+\frac{\left(m_{0}+1\right)}{2}\left(f_{0} f_{1}^{\prime \prime}+f_{1} f_{0}^{\prime \prime}\right)-2 m_{0} f_{0}^{\prime} f_{1}^{\prime}=-\frac{1}{2} f_{0} f_{0}^{\prime \prime}-\left(1-f_{0}^{\prime 2}\right)$, 


$$
\begin{aligned}
& \frac{1}{\sigma} h_{1}^{\prime \prime}+\frac{\left(m_{0}+1\right)}{2} f_{0} h_{1}^{\prime}-\left(2 m_{0}-1\right) f_{0}^{\prime} h_{1} \\
& =-a_{0}\left(\frac{1}{2} f_{0} \bar{h}_{0}^{\prime}+2 f_{0}^{\prime} \bar{h}_{0}\right)-a_{0} \frac{m_{0}+1}{2} f_{1} \bar{h}_{0}^{\prime}+a_{0}\left(2 m_{0}-1\right) f_{1}^{\prime} \bar{h}_{0},
\end{aligned}
$$

subject to

$f_{1}(0)=0, \quad f_{1}^{\prime}(0)=0, \quad h_{1}(0)=1, \quad f_{1}^{\prime} \rightarrow 0, h_{1} \rightarrow 0 \quad$ as $\eta \rightarrow \infty$.

Equation (14) is simply a perturbation to the Falkner-Skan solution at $m_{0}$, and our numerical integration gives $f_{1}^{\prime \prime}(0)=1.509195$. The left-hand side of Eq. (15) is essentially Eq. (12) and hence (15) has a complementary function $\bar{h}_{0}$ which satisfies homogeneous boundary conditions. Thus, we can specify $h_{1}^{\prime}(0)$ arbitrarily leading to a problem to determine $a_{0}$. We again used D02AGF in the NAG library to carry out this, finding $a_{0}=0.125381$, so that near the singularity at $m_{0}$ seen in Fig. $1 \mathrm{~b}$

$\theta^{\prime}(0) \sim 0.125381\left(m-m_{0}\right)^{-1}+\cdots$,

consistent with the plots shown in the figure.

\subsection{General case, $\lambda \neq 0$}

\section{$3.2 .1 m>0$}

We start by considering the case when $m>0$, where there is only a single solution to the Falkner-Skan (forced convection) problem, Fig. 1a. In Fig. 2, we plot $f^{\prime \prime}(0)$ and $\theta^{\prime}(0)$ against $\lambda$ for $m=1.0,0.5$ and 0.2 , noting that, for $m=0.2, \theta^{\prime}(0)=0$ for all $\lambda$. For these values of $m$, we see behaviour similar to that seen previously in mixed convection similarity solutions, see $[19,20,24,29,30]$, for example. There is a critical value $\lambda_{\mathrm{c}}$ of $\lambda$, with $\lambda_{\mathrm{c}}<0$, solutions only for $\lambda \geq \lambda_{\mathrm{c}}$, and a range of $\lambda$ where there are dual solutions. The values of $\lambda_{\mathrm{c}}$ depend on $m$, with $\left|\lambda_{\mathrm{c}}\right|$ decreasing as $m$ is decreased. The first solution branch (defined as the branch that contains the Falkner-Skan, $\lambda=0$ solution, as in Fig. 1a) continue to become large $\lambda$, not obvious from the plots in Fig. 2 but clearly seen in our numerical solutions.

We have already seen in the forced convection case that, at $m=0.2$, the sign of $\theta^{\prime}(0)$ changes from negative ( $m>0.2)$ to positive $(m<0.2)$. In Fig. 3, we plot $f^{\prime \prime}(0)$ and $\theta^{\prime}(0)$ against $\lambda$ for $m=0.1$. The picture for $f^{\prime \prime}(0)$ is similar to that seen in Fig. 2a, with a critical value $\lambda_{\mathrm{c}} \simeq-0.0347$, although being smaller than for $m=0.2$ (for which $\lambda_{\mathrm{c}} \simeq-0.31133$ ), with the first solution branch continuing to become large $\lambda$. However, now $\theta^{\prime}(0)>0$ gives rise to a temperature 'overshoot' for the first branch solutions and to large, positive values of $\theta^{\prime}(0)$ on the second solution branch, becoming increasingly larger as $\lambda \rightarrow 0$ from below.

Fig. 3 Plots of $f^{\prime \prime}(0)$ and $\theta^{\prime}(0)$ against $\lambda$ for $m=0.1$ and $\sigma=1.0$ obtained from the numerical solution of Eqs. (4-6)

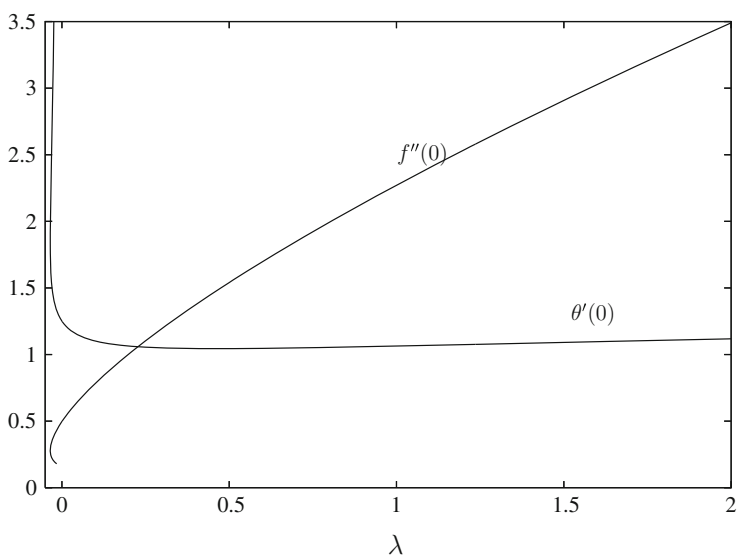


Fig. 4 Plots of a $f^{\prime \prime}(0)$ and b $\theta^{\prime}(0)$ against $\lambda$ for $m=0.02$ and $\sigma=1.0$ obtained from the numerical solutions of Eqs. (4-6). The first solution branch, defined as the branch that contains the Falkner-Skan, $\lambda=0$, solution, is shown by a broken line
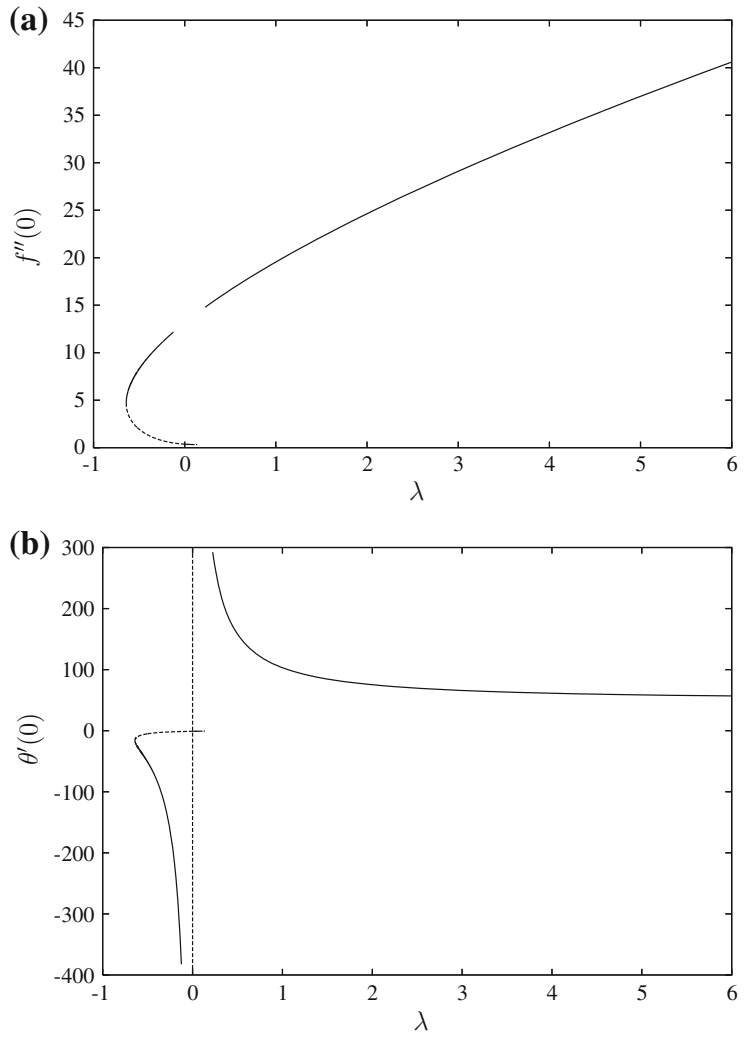

We have already identified a value $m_{0} \simeq 0.070722$ where the solution for forced convection becomes singular, Fig. 1b. This leads us to expect a similar situation in the general case and, to this end, we next consider a value of $m$ in the $0<m<m_{0}$ range. We choose $m=0.02$ as our example, and in Fig. 4, we again plot $f^{\prime \prime}(0)$ (Fig. 4a) and $\theta^{\prime}(0)$ (Fig. 4b) against $\lambda$. In these figures, the first solution branch (again defined as the branch that contains the Falkner-Skan solution) is shown by a broken line. This branch continues in a small way into the aiding-flow regime, i.e. $\lambda>0$, terminating at $\lambda=\lambda_{0} \simeq 0.1411$ in an apparently smooth fashion as appears from our numerical solution. There is a critical value $\lambda_{\mathrm{c}} \simeq-0.6410$, somewhat larger than that seen for $m=0.2$ and $m=0.1$, giving another (second) solution branch (full line). There is a range of $\lambda>\lambda_{\mathrm{c}}$ with dual solutions, and it is now the second solution branch that continues to become large $\lambda$. However, the behaviour of $\theta^{\prime}(0)$ is more like that seen in Fig. $1 \mathrm{~b}$ in that the second solution branch for $\theta$ becomes singular, now as $\lambda \rightarrow 0$ with $\left|\theta^{\prime}(0)\right| \rightarrow \infty$ although the solution for $\left.f^{\prime \prime}(0)\right)$ on this branch appears to pass smoothly through $\lambda=0$.

To determine the nature of this singularity on the second solution branch as $\lambda \rightarrow 0$, we put $\theta=\lambda^{-1} h$ and leave $f$ and $\eta$ unscaled. At leading order, we now have

$f^{\prime \prime \prime}+h+\frac{(m+1)}{2} f f^{\prime \prime}+m\left(1-f^{\prime 2}\right)=0, \quad \frac{1}{\sigma} h^{\prime \prime}+\frac{(m+1)}{2} f h^{\prime}-(2 m-1) f^{\prime} h=0$,

subject to

$f(0)=f^{\prime}(0)=0, h(0)=0, \quad f^{\prime} \rightarrow 1, h \rightarrow 0 \quad$ as $\eta \rightarrow \infty$.

In Fig. 5, we plot the values of $f^{\prime \prime}(0)$ and $h^{\prime}(0)$ obtained from the numerical solution of Eqs. $(18,19)$, then with $\theta^{\prime}(0)=\lambda^{-1} h^{\prime}(0)$, noting that this gives $\theta^{\prime}(0)>0$ as $\lambda \rightarrow 0$ from above and $\theta^{\prime}(0)<0$ as $\lambda \rightarrow 0$ from below, consistent with Fig. 4.

We see that a nontrivial solution to $(18,19)$ exists, i.e. a solution which does not have $h \equiv 0$, only for $m<m_{0}$, with $f^{\prime \prime}(0)$ approaching the corresponding Falkner-Skan solution and $h^{\prime}(0)$ approaching zero as $m \rightarrow m_{0}$. The 
Fig. 5 The limit as $\lambda \rightarrow 0$ : plots of $f^{\prime \prime}(0)$ and $h^{\prime}(0)$ against $m$ obtained from the numerical solution of Eqs. $(18,19)$. The value of $m_{\mathrm{s}}$ is shown by a broken line

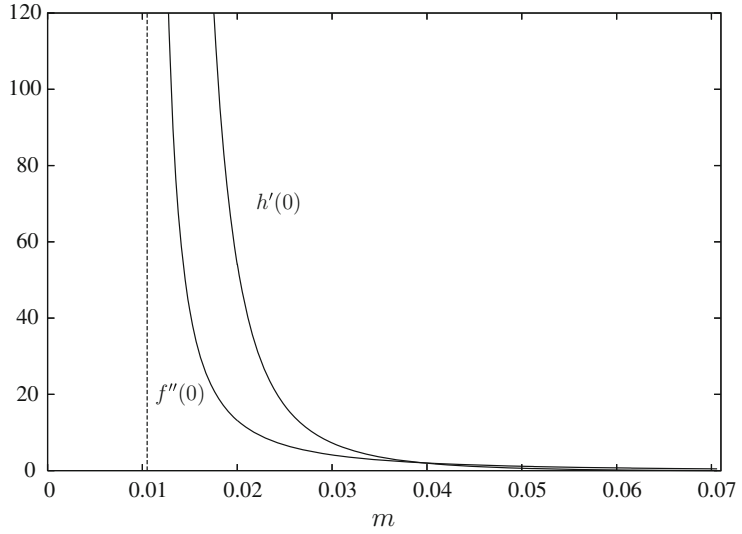

solution becomes singular, i.e. $f^{\prime \prime}(0)$ and $h^{\prime}(0)$ become large, as $m \rightarrow m_{\mathrm{s}}$ where $0<m_{\mathrm{s}}<m_{0}$. To see how the solution behaves as $m \rightarrow m_{\mathrm{s}}$, we put $m=m_{\mathrm{s}}+\delta$ and then

$f=\delta^{-1 / 2} \tilde{f}, h=\delta^{-2} \tilde{h}, \tilde{\eta}=\delta^{-1 / 2} \eta$.

When transformation $(20)$ is applied to Eqs. $(18,19)$, we obtain an expansion of the form:

$\tilde{f}(\tilde{\eta} ; \delta)=\tilde{f}_{0}(\tilde{\eta})+\delta \tilde{f}_{1}(\tilde{\eta})+\cdots \quad \tilde{h}(\tilde{\eta} ; \delta)=\tilde{h}_{0}(\tilde{\eta})+\delta \tilde{h}_{1}(\tilde{\eta})+\cdots$,

subject to the homogeneous boundary conditions in (19) and that now $\tilde{h}(0)=\delta^{2}, \tilde{f}^{\prime} \rightarrow \delta$ as $\tilde{\eta} \rightarrow \infty$ with primes denoting differentiation with respect to $\tilde{\eta}$. The leading-order problem is given by

$\tilde{f}_{0}^{\prime \prime \prime}+\tilde{h}_{0}+\frac{1}{2}\left(m_{\mathrm{s}}+1\right) \tilde{f}_{0} \tilde{f}_{0}^{\prime \prime}-m_{\mathrm{s}} \tilde{f}_{0}^{\prime 2}=0$,

$\frac{1}{\sigma} \tilde{h}_{0}^{\prime \prime}+\frac{1}{2}\left(m_{\mathrm{s}}+1\right) \tilde{f}_{0} \tilde{h}_{0}^{\prime}-\left(2 m_{\mathrm{s}}-1\right) \tilde{f}_{0}^{\prime} \tilde{h}_{0}=0$.

Since this is a homogeneous problem, we solve it subject to the extra condition $\tilde{f}^{\prime \prime}(0)=1$. It is the solution to this homogeneous problem, which determines $m_{\mathrm{s}}$ with our numerical integration again using D02AGF in the NAG library [28] gives $m_{\mathrm{s}}=0.010498$. In general, $\tilde{f}_{0}^{\prime \prime}(0)=a_{1}$ with the constant $a_{1}$ is determined by a consideration of the equations arising at $O(\delta)$. The details are not included here but are described below for the general problem. The limit $m_{\mathrm{s}}$ on the solution is indicated in Fig. 5 by a broken line. These results suggest that the solution for $\theta$ becomes singular as $\lambda \rightarrow 0$ in the way described above only for $m$ in the range $m_{\mathrm{s}}<m<m_{0}$.

Critical values, $\lambda_{\mathrm{c}}$ A significant feature of the results shown in Figs. 2, 3 and 4 is the existence of a critical value $\lambda_{\mathrm{c}}$ with dual solutions arising from the saddle-node bifurcation at $\lambda=\lambda_{\mathrm{c}}$. We now discuss these critical values in more detail. We can calculate $\lambda_{c}$ numerically following the approach given in [25,31], for example, whereby we make a linear perturbation to Eqs. (4-6) resulting in a linear homogeneous problem for this perturbation. To obtain this, we put $f=f_{\mathrm{c}}+\phi, \theta=\theta_{\mathrm{c}}+g$, where $\left(f_{\mathrm{c}}, \theta_{\mathrm{c}}\right)$ is the solution at the critical value and $\phi, g$ are small perturbations, which satisfy, on linearizing

$\phi^{\prime \prime \prime}+\frac{(m+1)}{2}\left(f_{\mathrm{c}} \phi^{\prime \prime}+f_{\mathrm{c}}^{\prime \prime} \phi\right)-2 m f_{\mathrm{c}}^{\prime} \phi^{\prime}+\lambda_{\mathrm{c}} g=0$,

$\frac{1}{\sigma} g^{\prime \prime}+\frac{(m+1)}{2}\left(f_{\mathrm{c}} g^{\prime}+\phi \theta_{\mathrm{c}}^{\prime}\right)-(2 m-1)\left(f_{\mathrm{c}}^{\prime} g+\phi^{\prime} \theta_{\mathrm{c}}\right)=0$,

$\phi(0)=\phi^{\prime}(0)=g(0)=0, \quad \phi^{\prime} \rightarrow 0, \quad g \rightarrow 0$ as $\eta \rightarrow \infty$ and $\phi^{\prime \prime}(0)=1$,

with the final condition in (25) being applied to force a nontrivial solution. When $\lambda_{\mathrm{c}}=0$, Eqs. $(23,24)$ have the solution $\phi=-m f_{\mathrm{c}}^{\prime}, g=-m \theta_{\mathrm{c}}^{\prime}$. This satisfies all the required boundary conditions in (25) apart from $\phi^{\prime}(0)=0, g(0)=0$, and satisfying this gives $f_{\mathrm{c}}^{\prime \prime}(0)=0, \theta_{\mathrm{c}}^{\prime}(0)=0$ as noted above for forced convection. 
Fig. 6 Plots of a the critical values $\lambda_{\mathrm{c}}$ for $m>0$ and $\mathbf{b} f_{\mathrm{c}}^{\prime \prime}(0), \mathbf{c} \theta_{\mathrm{c}}^{\prime}(0)$, their values at the critical point $\lambda_{\mathrm{c}}$, against $m$ for $\sigma=1.0$. The singularity at $m=m_{0}$ is shown by a broken line
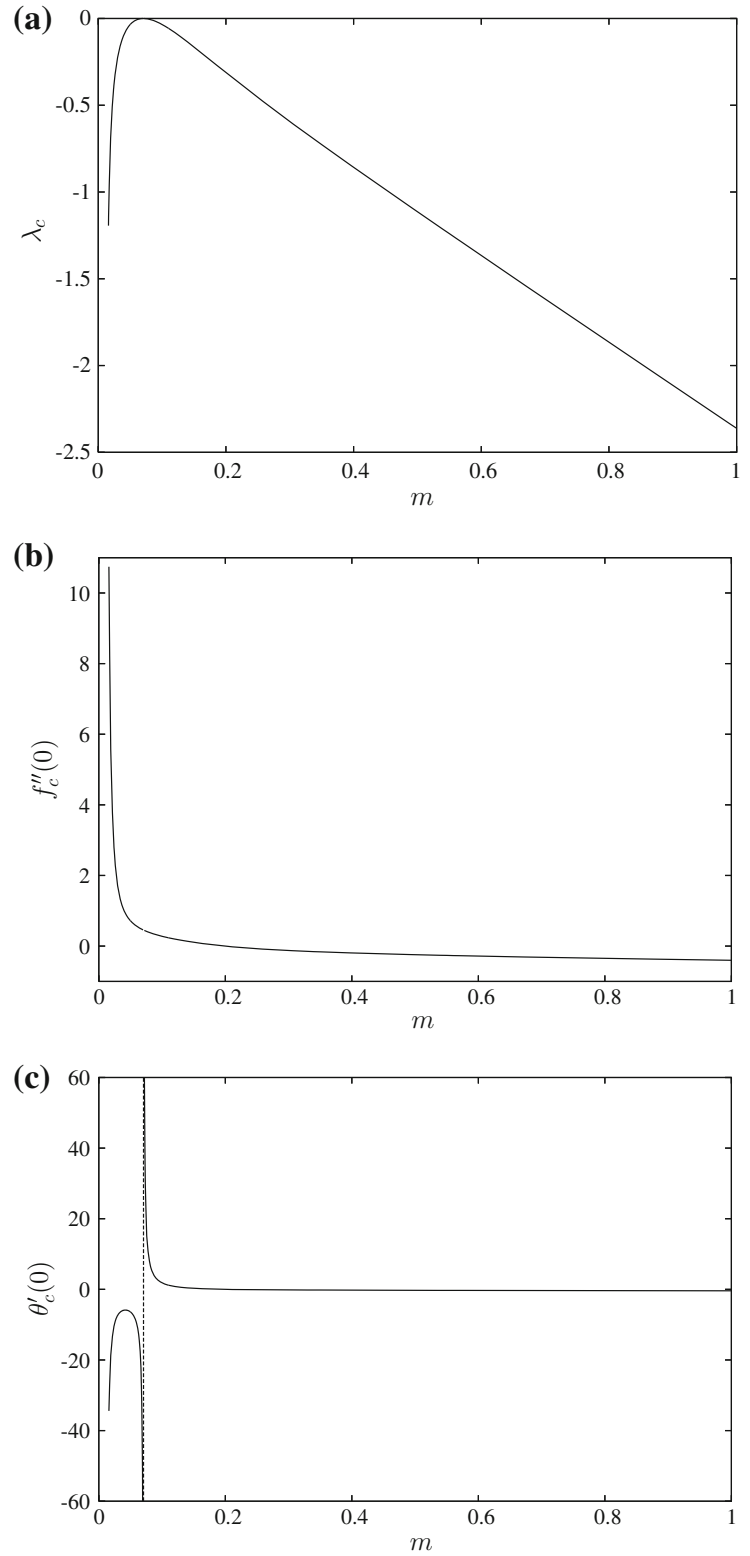

By obtaining a nontrivial solution to the eigenvalue problem (23-25), the values of $\lambda_{\mathrm{c}}$ are determined. In Fig. 6a, we plot $\lambda_{\mathrm{c}}$ against $m$, for $m>0$, obtained by this method. We see that $\lambda_{\mathrm{c}}$ decreases almost linearly for the larger values of $m$, and increases to zero at $m=m_{0}$ before decreasing again, becoming large as $\lambda \rightarrow 0$. In Fig. 6b, c, we plot $f_{\mathrm{c}}^{\prime \prime}(0)$ and $\theta_{\mathrm{c}}^{\prime}(0)$, respectively, the values of $f^{\prime \prime}(0)$ and $\theta^{\prime}(0)$ at $\lambda=\lambda_{\mathrm{c}}$. We see that $f_{\mathrm{c}}^{\prime \prime}(0)$ is negative for the larger values of $m$, changes sign at $m=0.2$, becoming large as $m \rightarrow 0$. To see why $f_{\mathcal{c}}^{\prime \prime}(0)=0$ at $m=\frac{1}{5}$, we have $(\phi, g)=-\left(\frac{1}{5}+\lambda_{\mathrm{c}}\right)\left(f_{\mathrm{c}}^{\prime}, \theta_{\mathrm{c}}^{\prime}\right)$ on noting that $\theta_{\mathrm{c}}^{\prime}(0)=0$ for this value of $m$. This satisfies all the boundary conditions in $(25)$ provided $f_{\mathrm{c}}^{\prime \prime}(0)=0$.

There is a singularity in the solution for $\theta_{\mathrm{c}}$ at $m=m_{0}$ (shown in Fig. $6 \mathrm{c}$ by a broken line), as perhaps might be expected from the forced convection solution, with $\theta_{\mathrm{c}}^{\prime}(0)>0$ becoming large as $m$ approaches $m_{0}$ from above. For $0<m<m_{0}, \theta_{\mathrm{c}}^{\prime}(0)<0$ also becoming large as $m \rightarrow m_{0}$ from below, compare Fig. $6 \mathrm{c}$ with Fig. $1 \mathrm{~b}$. The values of $\theta_{\mathrm{c}}^{\prime}(0)$ also become large and negative as $m \rightarrow 0$, in line with the results plotted in Fig. 4. 
Solution for large $\lambda$ : the free convection limit We have observed that a feature of the solutions described above is that, for $m>m_{0}$, the first solution branch and, for $0<m<m_{0}$, the second solution branch continue to large values of $\lambda$, and we now derive a solution valid in this limit. To do so, we put, following [32,33], for example, $f=\lambda^{1 / 4} F, \zeta=\lambda^{1 / 4} \eta$, thus leaving $\theta$ unscaled. When we apply this transformation to Eqs. (4-6) and then let $\lambda \rightarrow \infty$, we obtain, for the leading-order problem $\left(F_{0}, \theta_{0}\right)$, with

$F_{0}^{\prime \prime \prime}+\theta_{0}+\frac{m+1}{2} F_{0} F_{0}^{\prime \prime}-m F_{0}^{\prime 2}=0, \quad \frac{1}{\sigma} \theta_{0}^{\prime \prime}+\frac{m+1}{2} F_{0} \theta_{0}^{\prime}-(2 m-1) F_{0}^{\prime} \theta_{0}=0$,

subject to

$F_{0}(0)=F_{0}^{\prime}(0)=0, \quad \theta_{0}(0)=1, \quad F_{0}^{\prime} \rightarrow 0, \quad \theta_{0} \rightarrow 0 \quad$ as $\zeta \rightarrow \infty$,

where primes now denote differentiation with respect to $\zeta$.

In Fig. 7, we plot $F_{0}^{\prime \prime}(0)$ and $\theta_{0}^{\prime}(0)$ against $m$ obtained from the numerical solutions of $(26,27)$. We see that $F_{0}^{\prime \prime}(0)>$ 0 initially increases relatively slowly as $m$ is decreased from $m=1$ (the upper limit on $m$ used for the figure), then becoming large relatively quickly as $m$ is decreased further. Solutions were obtained for larger values of $m$ used in the figure. Also $\theta_{0}^{\prime}(0)$ is negative for larger $m$, changes sign at $m=0.2$, as expected, again becoming large and positive as $m$ becomes smaller. We note that our numerical solution of Eqs. $(26,27)$ did not appear to encounter any problem in passing through $m=m_{0}$. For $m$ large, we put $F_{0}=m^{-3 / 4} \bar{F}_{0}, \theta_{0}=\bar{\theta}_{0}, \bar{\zeta}=m^{1 / 4} \zeta$. The leading-order problem, obtained by substituting this transformation into Eqs. $(26,27)$ and letting $m \rightarrow \infty$, has the solution, for $\sigma=1.0$, $\bar{F}_{0}^{\prime \prime}(0)=0.71600, \bar{\theta}_{0}^{\prime}(0)=-0.69251$, giving $F_{0}^{\prime \prime}(0) \sim 0.71600 m^{-1 / 4}, \theta_{0}^{\prime}(0) \sim-0.69251 m^{1 / 4}$ as $m \rightarrow \infty$.

We then have

$f^{\prime \prime}(0) \sim \lambda^{3 / 4} F_{0}^{\prime \prime}(0)+\cdots, \quad \theta^{\prime}(0) \sim \lambda^{1 / 4} \theta_{0}^{\prime}(0)+\cdots \quad$ as $\lambda \rightarrow \infty$.

These results are consistent with our numerical solutions, giving positive values for $f^{\prime \prime}(0)$ throughout, as seen in Figs. 2a, 3 and 4a, whereas (28) gives $\theta^{\prime}(0)<0$ for $m>0.2$ (first solution branch), Fig. 2b and $\theta^{\prime}(0)>0$ for $0<m<0.2$, Figs. 3 and 4 b (second solution branch).

Although no problem was encountered at $m=m_{0}$, our numerical results did break down at a positive value $m_{\mathrm{i}}$ of $m$, with $0<m_{\mathrm{i}}<m_{0}$. To determine the value of $m_{\mathrm{i}}$ and to describe how the solution to $(26,27)$ behaves as $m \rightarrow m_{\mathrm{i}}$, we put $m=m_{\mathrm{i}}+\delta$, where $0<\delta \ll 1$ and then make the transformation:

$F_{0}=\delta^{-1 / 4} G, \quad \theta_{0}=\delta^{-1} H, \quad \xi=\delta^{-1 / 4} \zeta$.

Applying transformation (29) in $(26,27)$ gives

$G^{\prime \prime \prime}+H+\frac{1}{2}\left(m_{\mathrm{i}}+1+\delta\right) G G^{\prime \prime}-\left(m_{\mathrm{i}}+\delta\right) G^{\prime 2}=0$,

$\frac{1}{\sigma} H^{\prime \prime}+\frac{1}{2}\left(m_{\mathrm{i}}+1+\delta\right) G H^{\prime}-\left(2 m_{\mathrm{i}}-1+2 \delta\right) G^{\prime} H=0$,

now subject to

$G(0)=G^{\prime}(0)=0, \quad H(0)=\delta, \quad G^{\prime} \rightarrow 0, \quad H \rightarrow 0 \quad$ as $\xi \rightarrow \infty$,

primes now denoting differentiation with respect to $\xi$.

Equations (30-32) suggest looking for a solution valid for $\delta$ small by expanding

$G(\xi)=G_{0}(\xi)+\delta G_{1}(\xi)+\cdots, \quad H(\xi)=H_{0}(\xi)+\delta H_{1}(\xi)+\cdots$.

The leading-order problem is

$G_{0}^{\prime \prime \prime}+H_{0}+\frac{1}{2}\left(m_{\mathrm{i}}+1\right) G_{0} G_{0}^{\prime \prime}-m_{\mathrm{i}} G_{0}^{\prime 2}=0, \quad \frac{1}{\sigma} H_{0}^{\prime \prime}+\frac{1}{2}\left(m_{\mathrm{i}}+1\right) G_{0} H_{0}^{\prime}-\left(2 m_{\mathrm{i}}-1\right) H_{0} G_{0}^{\prime}=0$, 
subject to homogeneous boundary conditions arising from (32). As in the forced convection case, this is an eigenvalue problem for $m_{\mathrm{i}}$ and to determine $m_{\mathrm{i}}$, we construct the particular solution: $\left(\bar{G}_{0}, \bar{H}_{0}, \bar{\xi}\right)$ which has $\bar{G}_{0}^{\prime \prime}(0)=1$. We note that (34) is essentially the same problem given by (22) and our numerical integration for $\sigma=1$, again using D02AGF in the NAG library [28], gives $m_{\mathrm{i}}=0.010498$ and $\bar{H}_{0}^{\prime}(0)=0.77292$ as before. In general, $G_{0}^{\prime \prime}(0)=b_{0}$, with $b_{0}>0$, and putting $G_{0}=b_{0}^{1 / 3} \bar{G}_{0}, H_{0}=b_{0}^{4 / 3} \bar{H}_{0}, \bar{\xi}=b_{0}^{1 / 3} \xi$ leaves the leading-order problem unchanged.

To determine the constant $b_{0}$, we need to consider the terms of $O(\delta)$, and at this order,ifvujyh we have, on writing $G_{1}=b_{0}^{-1} \bar{G}_{1}$,

$\bar{G}_{1}^{\prime \prime \prime}+H_{1}+\frac{1}{2}\left(m_{\mathrm{i}}+1\right)\left(\bar{G}_{1} \bar{G}_{0}^{\prime \prime}+\bar{G}_{0} \bar{G}_{1}^{\prime \prime}\right)-2 m_{\mathrm{i}} \bar{G}_{0}^{\prime} \bar{G}_{1}^{\prime}=b_{0}^{4 / 3}\left(\bar{G}_{0}^{2}-\frac{1}{2} \bar{G}_{0} \bar{G}_{0}^{\prime \prime}\right)$,

$\frac{1}{\sigma} H_{1}^{\prime \prime}+\frac{1}{2}\left(m_{\mathrm{i}}+1\right)\left(\bar{G}_{0} H_{1}^{\prime}+\bar{G}_{1} \bar{H}_{0}^{\prime}\right)-\left(2 m_{\mathrm{i}}-1\right)\left(\bar{G}_{0}^{\prime} H_{1}+\bar{G}_{1}^{\prime} \bar{H}_{0}\right)=b_{0}^{4 / 3}\left(2 \bar{G}_{0}^{\prime} \bar{H}_{0}-\frac{1}{2} \bar{G}_{0} \bar{H}_{0}^{\prime}\right)$,

subject to $H_{1}(0)=1$, with primes denoting differentiation with respect to $\bar{\xi}$. Since the problem given by (35, 36) has a complementary function, namely $\bar{G}_{1}=\bar{\xi} \bar{G}_{0}^{\prime}+\bar{G}_{0}, H_{1}=\bar{\xi} \bar{H}_{0}^{\prime}+4 \bar{H}_{0}$, which satisfies homogeneous boundary conditions, this problem then leads us to determine $b_{0}$. Our numerical integration, again for $\sigma=1$, gives $b_{0}=0.18994$. Using this value in transformation (29) then gives

$F_{0}^{\prime \prime}(0) \sim 0.18994\left(m-m_{\mathrm{i}}\right)^{-3 / 4}+\cdots, \quad \theta_{0}^{\prime}(0) \sim 0.04851\left(m-m_{\mathrm{i}}\right)^{-5 / 4}+\cdots \quad$ as $m \rightarrow m_{\mathrm{i}}$.

Asymptotic expressions (37) are shown in Fig. 7 by broken lines and are in good agreement with the numerically determined values for $m$ close to $m_{\mathrm{i}}$.

The discussion given above and the results shown in Figs. 5c, 6 and 7 suggest that the solution becomes singular as $m \rightarrow 0$ which leads us to consider the case when $m=0$.

\section{$3.2 .2 m=0$}

The solution for $\lambda=0$ is the Blasius problem, see [3] for example. In Fig. 8, we plot $f^{\prime \prime}(0)$ and $\theta^{\prime}(0)$ against $\lambda$ for this case. We now find that the first solution branch can proceed only a small way into the aiding-flow regime, i.e. $\lambda>0$. There is a critical value at $\lambda=\lambda_{\mathrm{c}} \simeq 0.4947$ giving a range of $\lambda<\lambda_{\mathrm{c}}$ where there are dual solutions. The second solution branch terminates as $\lambda \rightarrow 0$, with $f^{\prime \prime}(0)$ appearing to approach a finite value and $\theta^{\prime}(0)$ becoming large. Here, as opposed to the previous cases for $m>0$, the first solution branch continues to large negative values of $\lambda$, i.e. there is a solution existing for the whole opposing-flow regime, i.e. $\lambda<0$.

To obtain the asymptotic solution as $\lambda \rightarrow-\infty$, we proceed as above, now putting $f=|\lambda|^{1 / 4} F, \zeta=|\lambda|^{1 / 4} \eta$ and again leaving $\theta$ unscaled. This leads to, for a general value of $m$, the leading-order problem $\left(F_{0}, \theta_{0}\right)$ satisfying

$F_{0}^{\prime \prime \prime}-\theta_{0}+\frac{m+1}{2} F_{0} F_{0}^{\prime \prime}-m F_{0}^{\prime 2}=0, \quad \frac{1}{\sigma} \theta_{0}^{\prime \prime}+\frac{m+1}{2} F_{0} \theta_{0}^{\prime}-(2 m-1) F_{0}^{\prime} \theta_{0}=0$,

subject to

$F_{0}(0)=F_{0}^{\prime}(0)=0, \quad \theta_{0}(0)=1, \quad F_{0}^{\prime} \rightarrow 0, \quad \theta_{0} \rightarrow 0 \quad$ as $\zeta \rightarrow \infty$,

where primes still denote differentiation with respect to $\zeta$.

In Fig. 9, we plot $F_{0}^{\prime \prime}(0)$ and $\theta_{0}^{\prime}(0)$ against $m$ obtained from the numerical solution of Eqs. $(38,39)$. The numerical solution shows the existence of a critical value $m_{\mathrm{a}} \simeq-0.074$ of $m$, with dual solutions for a range of values $m>m_{\mathrm{a}}$. The upper solution branch (as seen in Fig. 9a) becomes singular as $m \rightarrow m_{\mathrm{i}}$, indicated by broken lines, where $m_{\mathrm{i}} \simeq 0.010498$ is the same value given above. To see why this is the case, we can still apply transformation (29) to Eq. (38) with the only difference being that the sign of $H$ is changed in Eq. (30). The leading-order problem, which satisfies homogeneous boundary conditions, is essentially the same as (34), and hence leads to the same value for $m_{\mathrm{i}}$. Again, at $O(\delta)$, the only difference is a change in sign of $H_{1}$ in (35) leading to expressions (37) with the sign of $\theta^{\prime}(0)$ being negative. The lower solution branch terminates at $m \simeq 0.1744$ with both $F_{0}^{\prime \prime}(0)$ and $\theta_{0}^{\prime}(0)$ approaching the finite values of approximately -1.0734 and -0.4960 , respectively. The numerical solution indicates a weak 

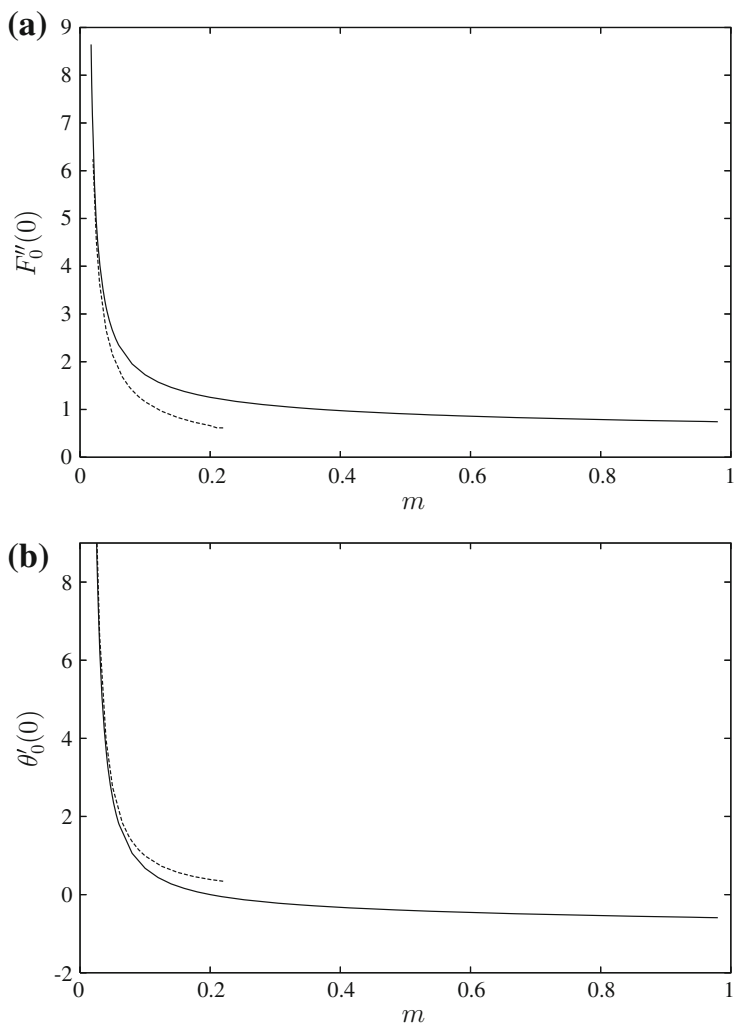

Fig. 7 The free convection limit, $\lambda \gg 1$, plots of a $F_{0}^{\prime \prime}(0)$ and b $\theta_{0}^{\prime}(0)$ against $m$ for $\sigma=1.0$, obtained from the numerical solution of Eqs. $(26,27)$. Asymptotic expressions (37) are shown by broken lines
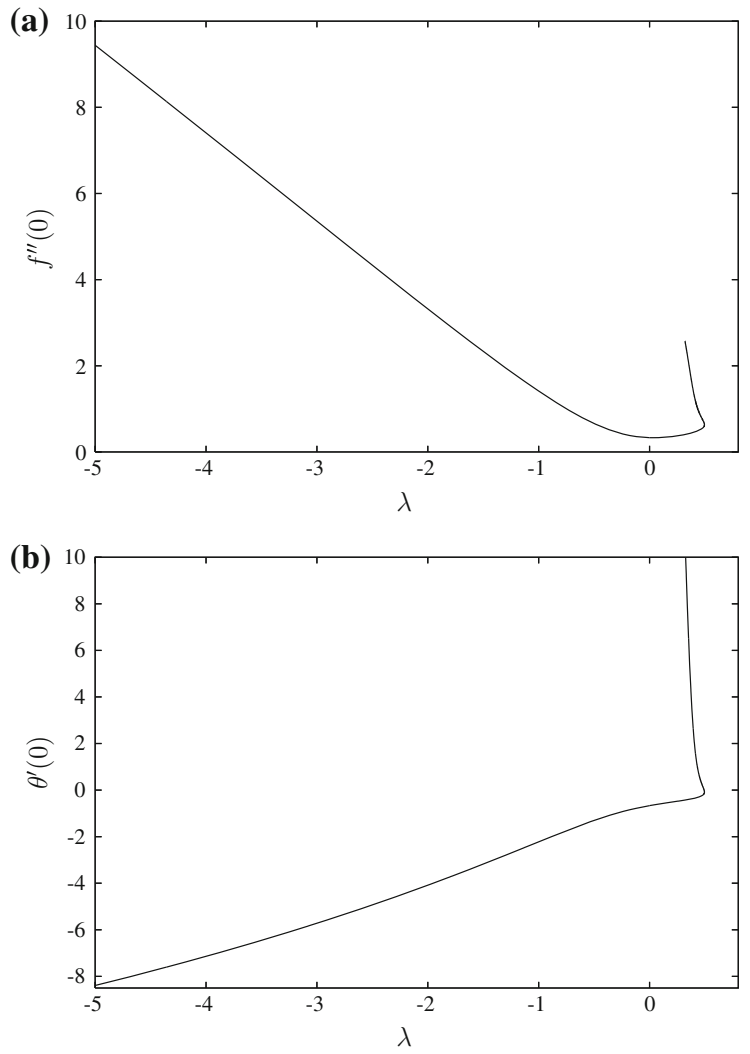

Fig. 8 Plots of $\mathbf{a} f^{\prime \prime}(0)$ and $\mathbf{b} \theta^{\prime}(0)$ against $\lambda$ for $m=0.0$ and $\sigma=1.0$ obtained from the numerical solution of Eqs. (4-6)

singularity as $m$ approaches this value, with the boundary-layer thickness increasing rapidly and the changes in $F_{0}^{\prime \prime}(0)$ and $\theta_{0}^{\prime}(0)$ increasing.

For $m=0$, we find that $F_{0}^{\prime \prime}(0) \simeq 5.3791, \theta_{0}^{\prime}(0) \simeq-14.6243$, giving

$f^{\prime \prime}(0) \sim 5.3791|\lambda|^{3 / 4}+\cdots, \quad \theta^{\prime}(0) \sim-14.6243|\lambda|^{1 / 4}+\cdots \quad$ as $\lambda \rightarrow-\infty \quad(m=0)$.

The values for $f^{\prime \prime}(0)$ and $\theta^{\prime}(0)$ given in (40) are relatively poor approximation to the results plotted in Fig. 8 and require much larger values of $|\lambda|$ to get better agreement (which is confirmed in our numerical integrations). This might be expected as the next order terms are only of $O\left(|\lambda|^{-1}\right)$.

$3.2 .3 m_{\mathrm{c}}<m<0$

In this case, for $0>m>m_{\mathrm{c}} \simeq-0.09043$, there are two solutions for the Falkner-Skan, $\lambda=0$, problem, see Fig. 1. In Fig. 10, we plot $f^{\prime \prime}(0)$ and $\theta^{\prime}(0)$ against $\lambda$ for $m=-0.05$ representative of this case. We see that there are now three solution branches, two arising from the saddle-node bifurcation at $\lambda_{\mathrm{c}} \simeq-0.1520$ contain the Falkner-Skan, $\lambda=0$, solution. The lower branch, which has $f^{\prime \prime}(0)<0$ at $\lambda=0$, terminates at a positive value of $\lambda$ with $f^{\prime \prime}(0)$ approaching zero and $\theta^{\prime}(0)$ approaching a finite negative value. There is a further saddle-node bifurcation on the middle solution branch, which has $f^{\prime \prime}(0)>0$ at $\lambda=0$, in the aiding-flow regime, i.e. at $\lambda_{\mathrm{c}} \simeq 0.5633$, giving a 

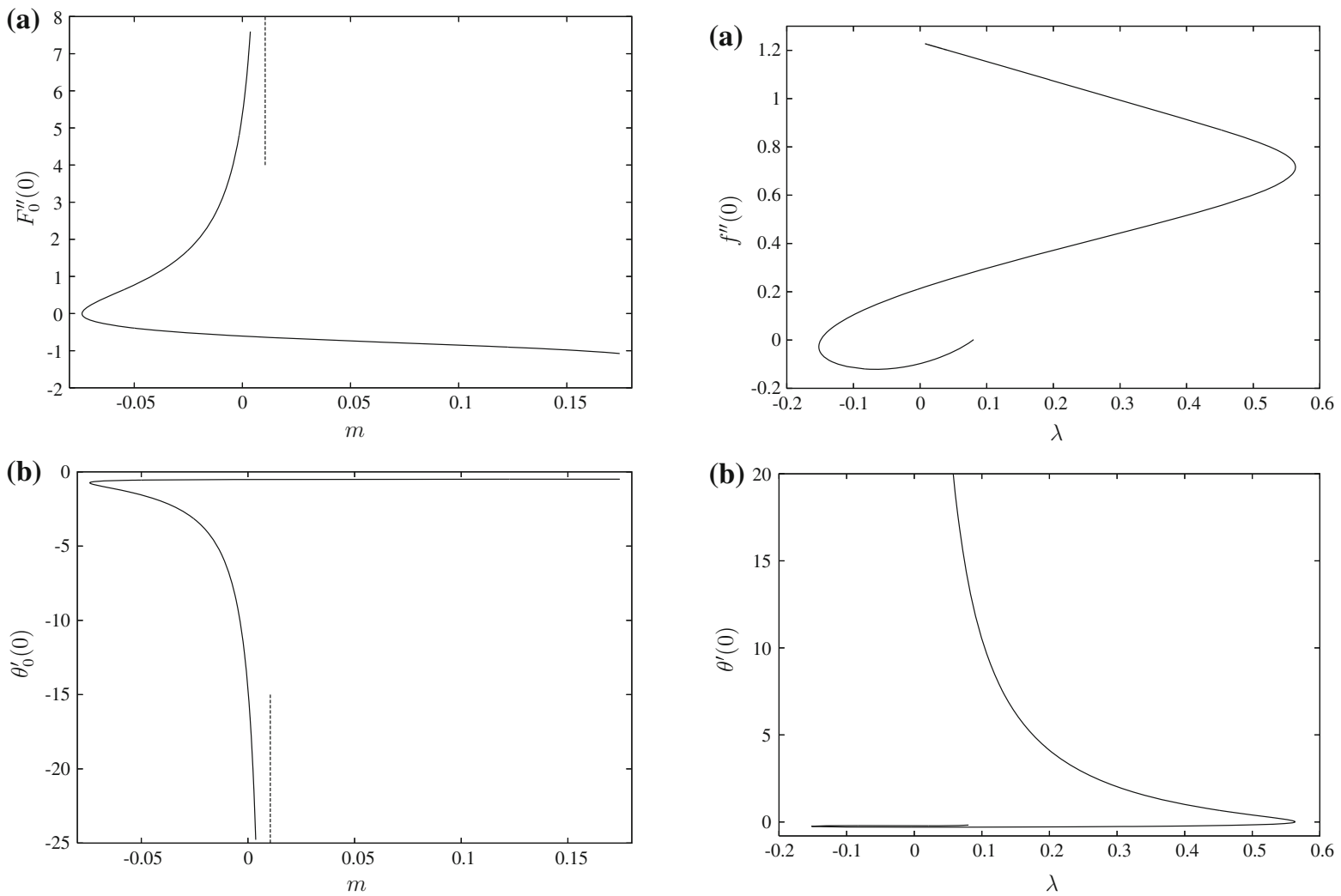

Fig. 9 The solution for $|\lambda| \gg 1, \quad \lambda<0$, plots of $\mathbf{a} F_{0}^{\prime \prime}(0)$ and $\mathbf{b}$ $\theta_{0}^{\prime}(0)$ against $m$ for $\sigma=1.0$, obtained from the numerical solution of Eqs. $(38,39)$

Fig. 10 Plots of a $f^{\prime \prime}(0)$ and $\mathbf{b} \theta^{\prime}(0)$ against $\lambda$ for $m=-0.05$ and $\sigma=1.0$ obtained from the numerical solution of Eqs. (4-6)

third solution branch. This upper solution arising from this saddle-node bifurcation terminates in a singularity as $\lambda \rightarrow 0$ from above, with $f^{\prime \prime}(0)$ approaching a finite positive value of approximately 1.2 and $\theta^{\prime}(0)$ becoming large.

The results shown in Fig. 10 do not proceed to large negative values of $\lambda$. However, we have already seen in Fig. 9 that solutions do exist in the limit as $\lambda \rightarrow-\infty$ for this value of $m$. In Fig. 11, we plot additional solutions for $m=-0.05$, with these solutions being disjoint from the solutions shown in Fig. 10. These solutions have a saddle-node bifurcation at $\lambda_{\mathrm{c}} \simeq-9.066$ with both solution branches proceeding to large negative values of $\lambda$. We can see why this is the case in Fig. 9. Here, for $m=-0.05$, there are are two solutions, one having $F_{0}^{\prime \prime}(0)=0.7695, \theta^{\prime}(0)=-1.5534$ and the other having $F_{0}^{\prime \prime}(0)=-0.3920, \theta^{\prime}(0)=-0.5541$, giving

$f^{\prime \prime}(0) \sim 0.7695|\lambda|^{3 / 4}+\cdots, \quad \theta^{\prime}(0) \sim-1.5534|\lambda|^{1 / 4}+\cdots$,

for the upper solution branch and

$f^{\prime \prime}(0) \sim-0.3920|\lambda|^{3 / 4}+\cdots, \quad \theta^{\prime}(0) \sim-0.5541|\lambda|^{1 / 4}+\cdots$,

for the lower solution branch as $\lambda \rightarrow \infty$, consistent with the plots shown in Fig. 11 .

Critical values We have seen the existence of critical values $\lambda_{\mathrm{c}}$ in the results for $m=0$ (Fig. 8) and for $m=-0.05$ (Fig. 10), and so the picture shown in Fig. 6 does not reveal the full story. To complete the picture, we now concentrate on values of $m \leq 0$ and in Fig. 12a, we plot further values of $\lambda_{\mathrm{c}}$ against $m$. Our numerical calculations, using Eqs. (23-25), give two disjoint branches. The upper branch terminates at $m \simeq 0.0229$ and at $m \simeq-0.1875$ and has $\lambda_{\mathrm{c}}>0$ with $\lambda_{\mathrm{c}}$ changing only relatively slowly in this range. The lower branch changes sign from negative to 
Fig. 11 Further plots of a $f^{\prime \prime}(0)$ and $\mathbf{b} \theta^{\prime}(0)$ against $\lambda$ for $m=-0.05$ and $\sigma=1.0$ obtained from the numerical solution of Eqs. (4-6)
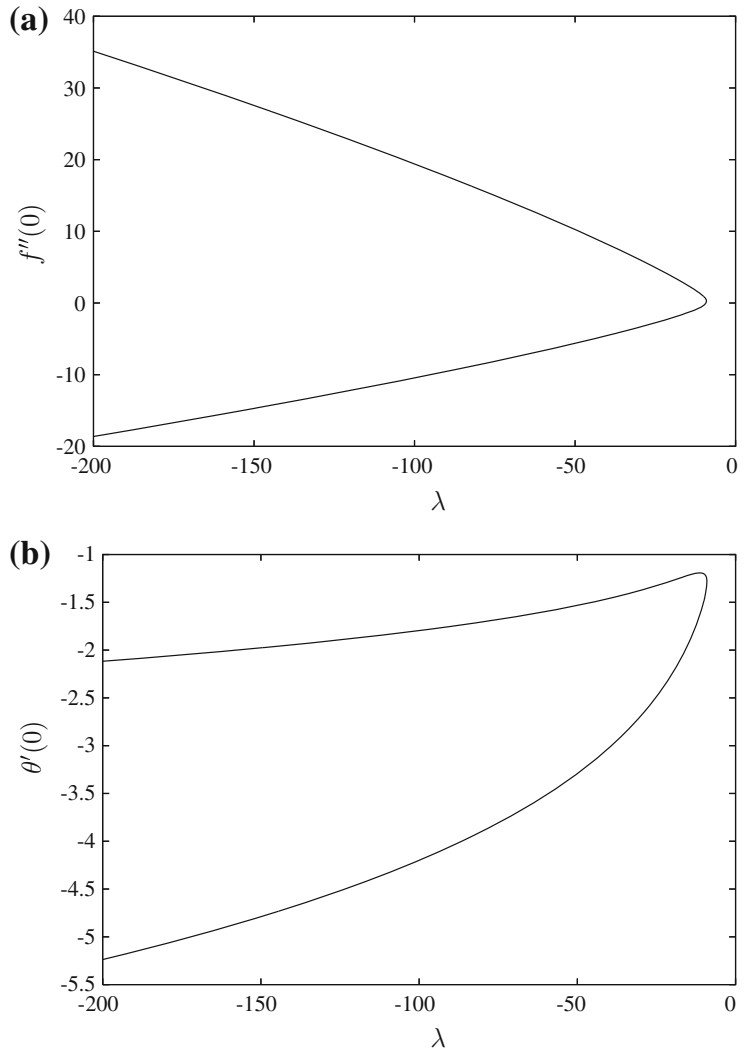

positive at $m \simeq-0.0904=m_{\mathrm{c}}$, approaches the large $|\lambda|$ limit of $m \simeq-0.074$ and terminates at $m \simeq-0.1875$. In Fig. $12 \mathrm{~b}$, c, we plot, respectively, $f_{\mathrm{c}}^{\prime \prime}(0)$ and $\theta_{\mathrm{c}}^{\prime}(0)$. The values of $f_{\mathrm{c}}^{\prime \prime}(0)$ on the upper branch have only relatively small variation across the whole range of $m$ and become singular at both ends of this range, more obvious in the figure at the upper end. The values of $f_{\mathrm{c}}^{\prime \prime}(0)$ on the lower branch show a slightly greater variation and again become singular at both ends of the range, now more obvious at the lower end. The values of $\theta_{c}^{\prime}(0)$ on the upper branch, shown in Fig. 12c, become large and positive at the lower end of the range and approach zero at the upper end. The values of $\theta_{\mathrm{c}}^{\prime}(0)$ on the lower branch show a relatively small variation and do not appear to become singular at either end.

\subsection{4 $m<m_{\mathrm{c}}$}

One feature to emerge from Fig. 12 is the existence of solutions for $m<m_{\mathrm{c}}$, i.e. where there are no solutions in the Falkner-Skan, $\lambda=0$, problem. To consider this situation, we took a value of $m=-0.15<m_{\mathrm{c}}$, where we see a critical value $\lambda_{\mathrm{c}}$, Fig. 12a, and in Fig. 13 we plot $f^{\prime \prime}(0)$ and $\theta^{\prime}(0)$ against $\lambda$. We find a critical value $\lambda_{\mathrm{c}} \simeq 0.3119$, consistent with Fig. 12 (upper branch), with dual solutions in $0<\lambda<\lambda_{\mathrm{c}}$ and with both solution branches becoming singular as $\lambda \rightarrow 0$ in which $f^{\prime \prime}(0)$ appears to be approaching constant values and $\theta^{\prime}(0)$ becoming large. (We note that $\theta^{\prime}(0)$ achieved much larger values on both solution branches as $\lambda$ became small than those that are used to plot Fig. 13). We were unable to find any solutions in the opposing-flow regime, i.e. $\lambda<0$.

Our discussion to present has concentrated on determining the behaviour of the solution for specific values of $m$, i.e essentially plots of $f^{\prime \prime}(0)$ and $\theta^{\prime}(0)$ against $\lambda$ for a given value of $m$. We can gain further insight into the nature of problem by fixing a value for $\lambda$ and considering how the solution varies with $m$. This is what we propose to do next. 
Fig. 12 Plots of a the critical values $\lambda_{\mathrm{c}}$ for $m<0$ and $\mathbf{b} f_{\mathrm{c}}^{\prime \prime}(0), \mathbf{c} \theta_{\mathrm{c}}^{\prime}(0)$, their values at the critical point $\lambda_{\mathrm{c}}$, against $m$ for $\sigma=1.0$ (a)
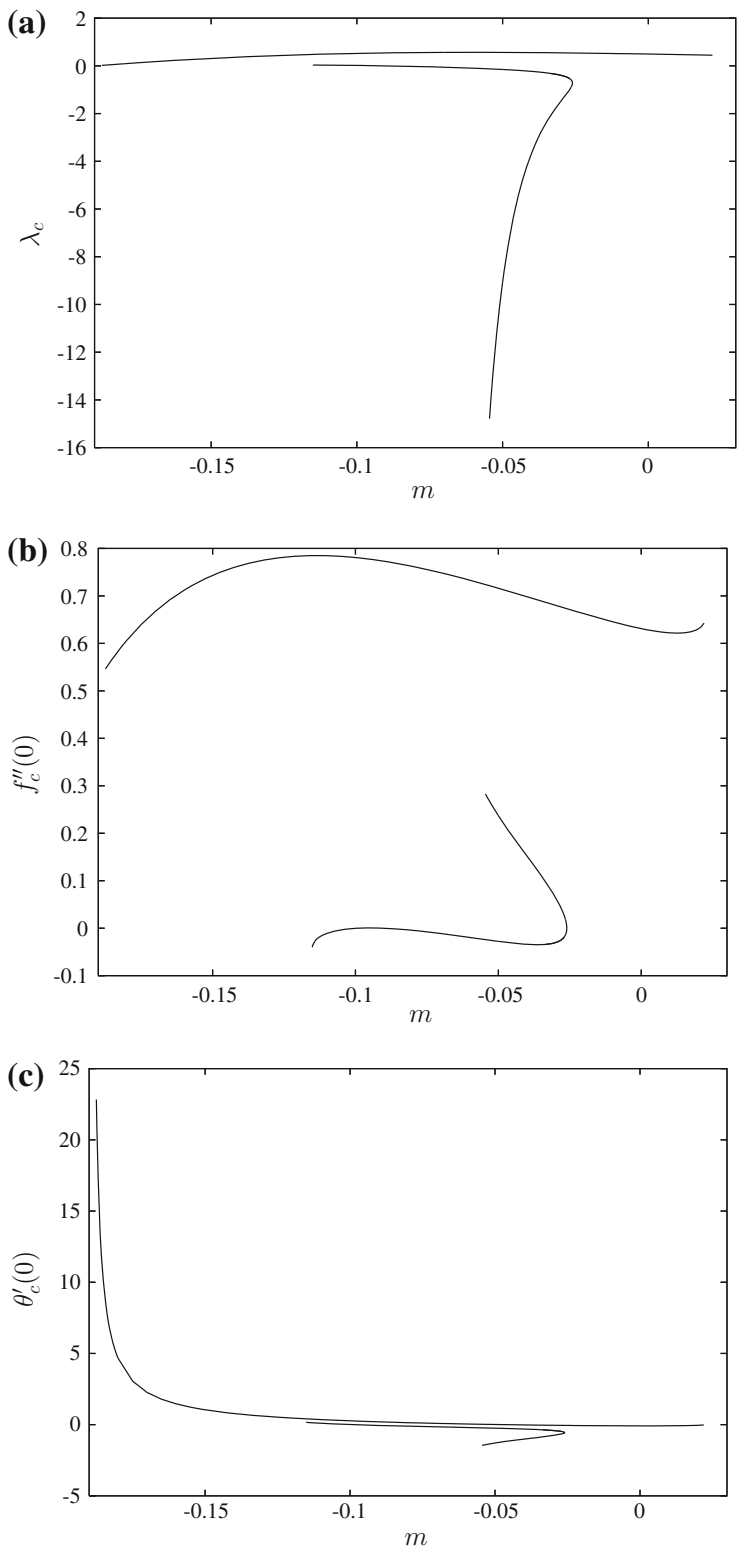

\subsection{Variation with $m$}

We consider two cases, $\lambda=0.5$, representative of aiding flow, and $\lambda=-0.5$, representative of opposing flow.

\subsubsection{Aiding flow}

In Fig. 14, we plot results for $\lambda=0.5$. For this case, there is no critical value in $m>0$, Fig. 6a, and only one critical value, at $m \simeq-0.1084$ in $m<0$, Fig. 12a. This is reflected in the results shown in Fig. 14 where we see two disjoint branches. The upper branch, $m>0$, continues to become large $m$ and becomes singular as $m$ approaches a small, but strictly positive, value $m_{\mathrm{s}}$, with both $f^{\prime \prime}(0)$ and $\theta^{\prime}(0)$ becoming large and positive and the boundary-layer thickness decreasing. The other solution branch exists only for a finite range of $m$ with the critical 

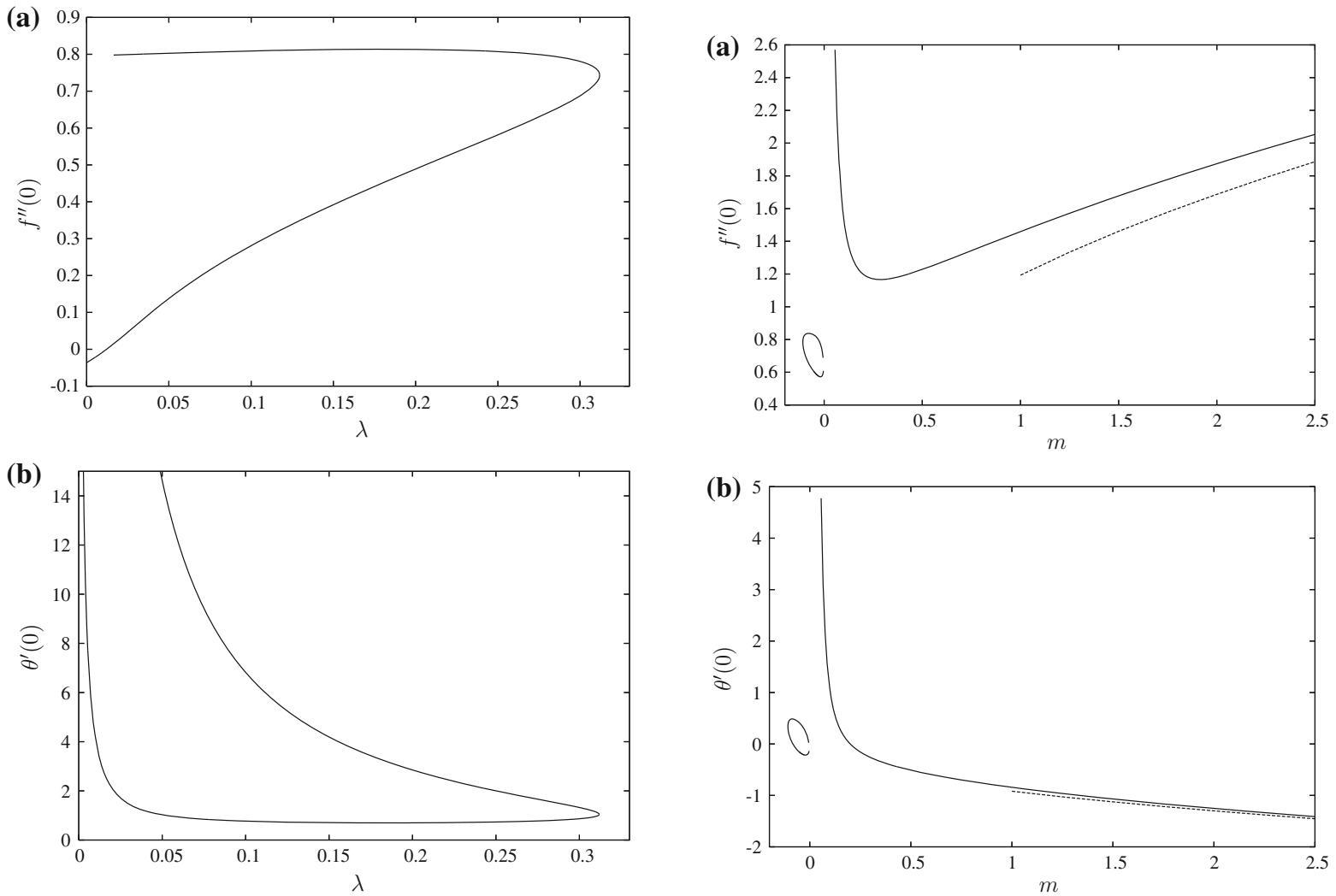

Fig. 13 Plots of a $f^{\prime \prime}(0)$ and $\mathbf{b} \theta^{\prime}(0)$ against $\lambda$ for $m=-0.15$ and $\sigma=1.0$ obtained from the numerical solution of Eqs. (4-6)

Fig. 14 Plots of a $f^{\prime \prime}(0)$ and $\mathbf{b} \theta^{\prime}(0)$ against $m$ for $\lambda=0.5$, representative of aiding flow, and $\sigma=1.0$ obtained from the numerical solution of Eqs. (4-6). The asymptotic expressions for large $m$ are shown by broken lines

value at $m=m_{\mathrm{c}} \simeq-0.1084$ producing dual solutions in $m_{\mathrm{c}}<m<0$. Both solution branches terminate as $m \rightarrow 0$ from below with both $f^{\prime \prime}(0)$ and $\theta^{\prime}(0)$ appearing to approach finite values though in a singular way with the boundary-layer thickness increasing as $m$ decreases to zero.

The solution for large $m$ follows that given above for forced convection. If we apply the transformation given, then we find that the buoyancy term enters the problem at leading order only if $\lambda$ is of $O(m)$. When $\lambda$ is of $O(1)$, the leading-order problem for $m$ large is given by Eq. (7) together with

$\frac{1}{\sigma} \theta^{\prime \prime}+\frac{1}{2} \bar{f} \theta^{\prime}-2 \bar{f}^{\prime} \theta=0, \quad \theta(0)=1, \quad \theta \rightarrow 0$ as $\bar{\eta} \rightarrow \infty$.

On solving Eq. (43), we find that $\theta^{\prime}(0) \sim-0.91925 m^{1 / 2}+\cdots$ as $m \rightarrow \infty$. This asymptotic result is shown in Fig. $14 \mathrm{~b}$ by a broken line and is in very good agreement with the numerically determined values even at relatively small values of $m$. The corresponding $f^{\prime \prime}(0) \sim 1.19304 m^{1 / 2}+\cdots$ is shown in Fig. 14a, again by a broken line, in agreement with the numerical values being not as good as for $\theta^{\prime}(0)$.

To determine the value of $m_{\mathrm{S}}$ and the nature of the solution as $m \rightarrow m_{\mathrm{s}}$, we follow an approach similar to that seen above in the free convection limit. We start by putting $m=m_{\mathrm{s}}+\delta$, where $0<\delta \ll 1$ and then put

$f=\delta^{-1 / 2} g, \quad \theta=\delta^{-2} \frac{h}{\lambda}, \quad Y=\delta^{-1 / 2} \eta \quad(\lambda \neq 0)$. 
When transformation (44) is substituted into Eqs. (4-6), an expansion of the form

$g(Y ; \delta)=g_{0}(Y)+\delta g_{1}(Y)+\cdots \quad h(Y ; \delta)=h_{0}(Y)+\delta h_{1}(Y)+\cdots$

is suggested with the leading-order term being given by

$g_{0}^{\prime \prime \prime}+h_{0}+\frac{1}{2}\left(m_{\mathrm{s}}+1\right) g_{0} g_{0}^{\prime \prime}-m_{\mathrm{s}} g_{0}^{\prime 2}, \quad \frac{1}{\sigma} h_{0}^{\prime \prime}+\frac{1}{2}\left(m_{\mathrm{s}}+1\right) g_{0} h_{0}^{\prime}-\left(2 m_{\mathrm{s}}-1\right) g_{0}^{\prime} h_{0}=0$,

subject to the homogeneous boundary conditions

$g_{0}(0)=0, \quad g_{0}^{\prime}(0)=0, \quad h_{0}(0)=0, \quad g_{0}^{\prime}, h_{0} \rightarrow 0$ as $Y \rightarrow \infty$,

where primes now denote differentiation with respect to $Y$. Equations $(46,47)$ represent an eigenvalue problem for $m_{\mathrm{s}}$ and and is the same as that given in (34) in the large $\lambda$ limit. As before, we determine that particular solution $\left(\bar{g}_{0}, \bar{h}_{0}, \bar{Y}\right)$ which has $\bar{g}_{0}^{\prime \prime}(0)=1$, and so, for $\sigma=1, m_{\mathrm{s}}=m_{\mathrm{i}}=0.010498$ with a corresponding value, $\bar{h}_{0}^{\prime}(0)=0.77292$. We note that this value for $m_{\mathrm{s}}$ is independent of $\lambda$. Now $g_{0}^{\prime \prime}(0)=d_{0}$, for some $d_{0}>0$, and the general solution is then given by

$g_{0}=d_{0}^{1 / 3} \bar{g}_{0}, \quad h_{0}=d_{0}^{4 / 3} \bar{h}_{0}, \quad \bar{Y}=d_{0}^{1 / 3} Y$.

When we apply (48) in the next order term in expansion (45) and put $g_{1}=d_{0}^{-1 / 3} \bar{g}_{1}, h_{1}=d_{0}^{2 / 3} \bar{h}_{1}$ we find that, at $O(\delta)$,

$\bar{g}_{1}^{\prime \prime \prime}+\bar{h}_{1}+\frac{1}{2}\left(m_{\mathrm{s}}+1\right)\left(\bar{g}_{0} \bar{g}_{1}^{\prime \prime}+\bar{g}_{0}^{\prime \prime} \bar{g}_{1}\right)-2 m_{\mathrm{s}} \bar{g}_{0}^{\prime} \bar{g}_{1}^{\prime}=d_{0}^{2 / 3}\left(\bar{g}_{0}^{\prime 2}-\frac{1}{2} \bar{g}_{0} \bar{g}_{0}^{\prime \prime}\right)$,

$\frac{1}{\sigma} \bar{h}_{1}^{\prime \prime}+\frac{1}{2}\left(m_{\mathrm{s}}+1\right)\left(\bar{g}_{0} \bar{h}_{1}^{\prime}+\bar{g}_{1} \bar{h}_{0}^{\prime}\right)-\left(2 m_{\mathrm{s}}-1\right)\left(\bar{g}_{0} \bar{h}_{1}^{\prime}+\bar{g}_{1}^{\prime} \bar{h}_{0}\right)=d_{0}^{2 / 3}\left(2 \bar{g}_{0} \bar{h}_{0}-\frac{1}{2} \bar{g}_{0} \bar{h}_{0}^{\prime}\right)$,

subject to $\bar{g}_{1}^{\prime} \rightarrow 1$ as $\bar{Y} \rightarrow \infty$. Since Eqs. $(49,50)$ have a complementary function $\left(\bar{Y} \bar{g}_{0}^{\prime}+\bar{g}_{0}, \bar{Y} \bar{h}_{0}^{\prime}+4 \bar{h}_{0}\right)$ which satisfies homogeneous boundary conditions, this becomes a problem to determine $d_{0}$. Our numerical integration gives, for $\sigma=1, d_{0}=0.01299$. From (44), we then have, for $\lambda=0.5$,

$f^{\prime \prime}(0) \sim 0.01299\left(m-m_{\mathrm{s}}\right)^{-3 / 2}+\cdots, \quad \theta^{\prime}(0) \sim 0.0011\left(m-m_{\mathrm{s}}\right)^{-5 / 2}+\cdots$ as $m \rightarrow m_{\mathrm{s}}$.

The values for $f^{\prime \prime}(0)$ and $\theta^{\prime}(0)$ given in (51) are in reasonable agreement with the numerical values plotted in Fig. 14 for $m$ close to $m_{\mathrm{s}}$.

\subsubsection{Opposing flow}

We plot the results for $\lambda=-0.5$ in Fig. 15. Here, from Figs. 6a and 12a, there are three critical values of $m$. The saddle-node bifurcation at $m \simeq 0.2660$ produces dual solutions, one branch of which continues to large $m$ in the way described above. The other branch terminates at a finite value of $m \simeq 0.3494$. There is another disjoint solution branch arising from the critical value at $m \simeq-0.0275$ giving one solution branch that continues into $m>0$, with another critical value at $m \simeq 0.0221$ (not obvious in the figure but seen clearly in the numerical results) and then again becoming singular as $m \rightarrow m_{\mathrm{s}} \simeq 0.01050$ from above, with $f^{\prime \prime}(0)$ and $\theta^{\prime}(0)$ becoming large and positive/negative. The behaviour of the solution close to $m_{\mathrm{s}}$ is similar to that given above for aiding flow, the only difference being a change of sign for $\theta$ resulting from (44) and the consequent change of sign for $\theta^{\prime}(0)$ in (51). The other other solution branch terminates at $m \simeq 0.1814$, so that it appears that, in this case, there is a finite range of $m$ over which there is no solution.

\section{Discussion and conclusions}

We have considered the effects of mixed convection on the classical Falkner-Skan system involving the two parameters, $m$ associated with the form of the outer flow and the mixed convection parameter $\lambda$. We have observed 

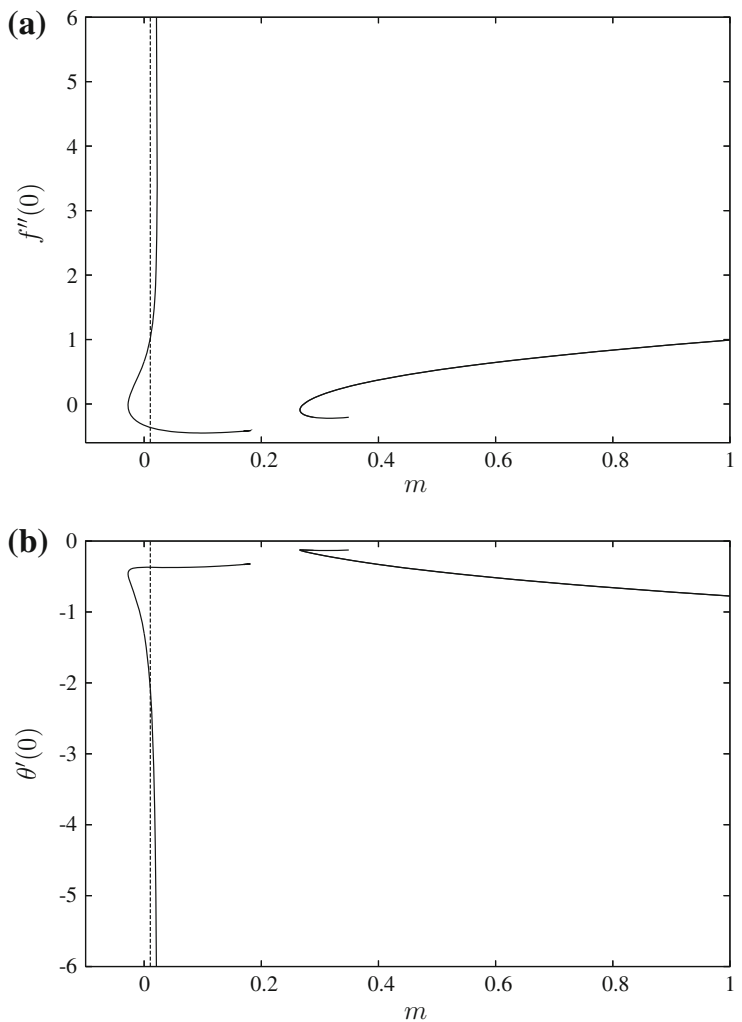

Fig. 15 Plots of a $f^{\prime \prime}(0)$ and $\mathbf{b} \theta^{\prime}(0)$ against $m$ for $\lambda=-0.5$, representative of opposing flow, and $\sigma=1.0$ obtained from the numerical solution of Eqs. (4-6). The singularity at $m=m_{\mathrm{s}}$ is indicated by a broken line
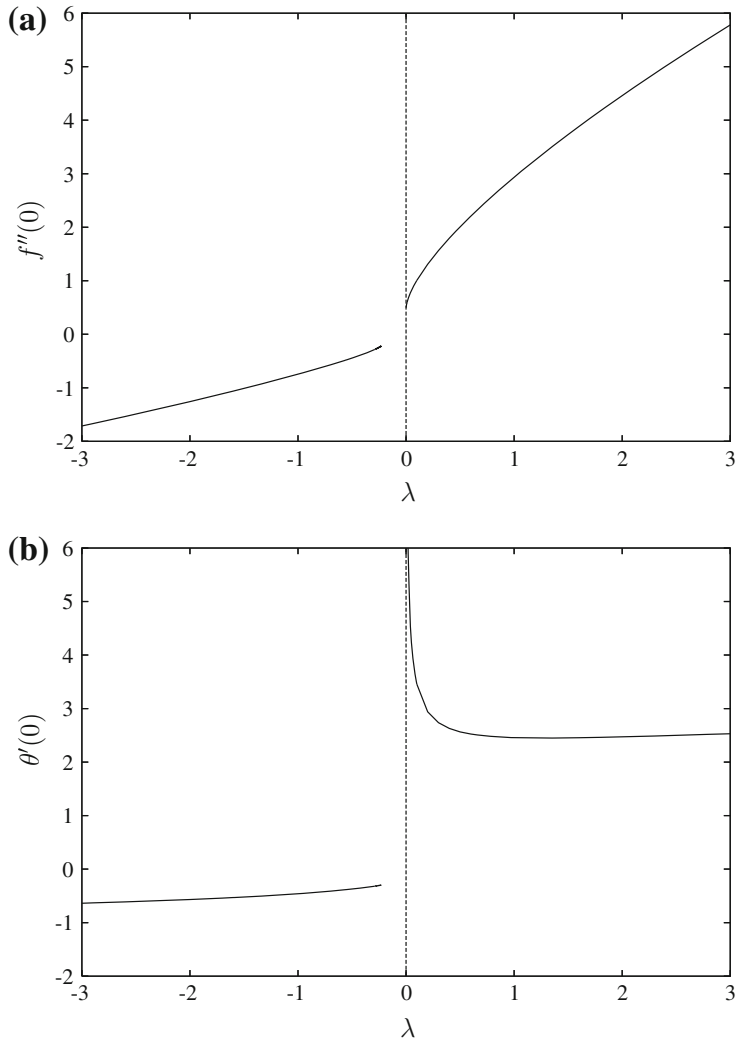

Fig. 16 Plots of a $f^{\prime \prime}(0)$ and $\mathbf{b} \theta^{\prime}(0)$ against $\lambda$ for $m=m_{0}=$ 0.070722 and $\sigma=1.0$ obtained from the numerical solution of Eqs. (4-6)

some, perhaps unexpected, results. The first of these is that, for forced convection, the existence of a value $m=$ $m_{0} \simeq 0.07072$ where there is a singularity in the solution for the temperature, Fig. 1b, this being independent of the flow. We consider this case further in Fig. 16 with plots of $f^{\prime \prime}(0)$ and $\theta^{\prime}(0)$ against $\lambda$ for $m=0.070722$. The singular nature of the solution as $\lambda \rightarrow 0$ can clearly be seen in the figure with $f^{\prime \prime}(0)$ approaching its Falkner-Skan value of approximately 0.4541 as $\lambda$ approaches zero from above. For $\lambda>0, \theta^{\prime}(0)$ becomes increasingly larger as $\lambda \rightarrow 0^{+}$, whereas for, $\lambda<0$, both $f^{\prime \prime}(0)$ and $\theta^{\prime}(0)$ approach finite values, although with the thickness of the boundary layer increasing, as $\lambda \rightarrow 0^{-}$. As can be seen in Figs. 7 and 9, the solution proceeds to both large positive and negative values of $\lambda$ in this case, with

$$
\begin{aligned}
& f^{\prime \prime}(0) \sim 2.143 \lambda^{3 / 4}+\cdots, \quad \theta^{\prime}(0) \sim 1.425 \lambda^{5 / 4}+\cdots \quad \text { as } \lambda \rightarrow \infty, \\
& f^{\prime \prime}(0) \sim-0.783|\lambda|^{3 / 4}+\cdots, \quad \theta^{\prime}(0) \sim-0.502|\lambda|^{5 / 4}+\cdots \quad \text { as } \lambda \rightarrow-\infty .
\end{aligned}
$$

We find that asymptotic expressions $(52,53)$ are not particularly good fit to the numerical values for the values of $\lambda$ used to plot Fig. 16.

For the general problem, we started by considering the case when $m>m_{0}$ finding behaviour similar to that seen previously in related problems, Figs. 2 and 3, in which there is a critical value $\lambda_{c}$ of $\lambda$, dependent on $m$, limiting the range of solution to $\lambda \geq \lambda_{\mathrm{c}}$ with one solution branch continuing to large positive values of $\lambda$ and the other branch terminating at a finite value of $\lambda$. We then took a value for $m$ in $0<m<m_{0}$, Fig. 4, finding similar behaviour, 
although it was now the first solution branch, i.e. that branch containing the Falkner-Skan, $\lambda=0$, solution, which terminated at a finite value of $\lambda$, whereas the second solution branch was now the one that continued to large $\lambda$.

This led us to consider the free convection, $\lambda \rightarrow \infty$, limit, Fig. 7. The main feature of this asymptotic solution was the existence of a value $m_{\mathrm{i}} \simeq 0.0105$ of $m$ limiting the possibility of having solution continuing to large $\lambda$ to only $m>m_{\mathrm{i}}$. This was reflected in the results for $m=0$, Fig. 8, which contains the classical Blasius solution for $\lambda=0$. Here the solution for aiding flow, $\lambda>0$, continued only to a saddle-node bifurcation at $\lambda \simeq 0.4947$ which gave two solution branches, the one containing the Blasius solution continued to become large negative values of $\lambda$. Following on from this, we next considered the solution for $|\lambda| \gg 1, \lambda<0$, Fig. 9, again that it it became singular as $m \rightarrow m_{\mathrm{i}}$. There was also a saddle-node bifurcation at $m=m_{\mathrm{a}} \simeq-0.074$ putting a lower bound on $m$ for a solution in this limit.

We then looked at the case when $m<0$, starting with a case when $m>m_{\mathrm{c}}$, Figs. 10 and 11, i.e. where there are two solutions in the original Falkner-Skan system. Here we found two separate parts to the solution, one part arising from the two solutions to the Falkner-Skan problem in $0>m>m_{\mathrm{c}}$ Fig. 10, and a completely separate part, Fig. 11, giving the two solutions that appear in the $|\lambda|$ limit. This gave three critical values, two in the first part and one in the second part, leading us to consider the critical values $\lambda_{\mathrm{c}}$ further for $m<0$ in Fig. 12. This figure reveals two disjoint branches, as might be expected from the results shown in Figs. 10 and 11. One branch continues the Falkner-Skan solution with $\lambda_{\mathrm{c}}=0$ and the other branch arises from the saddle-node bifurcation in the large $|\lambda|$ limit. It might appear from Fig. 12a that these two branches could join (although it is less obvious in Fig. 12b). However, we were unable to do so even when progressing the numerical solution in very small increments of $m$.

A feature of our calculations of $\lambda_{\mathrm{c}}$ was a range of $m<m_{\mathrm{c}}$ where a solution is indicated. We examined this case in Fig. 13 for $m=-0.15<m_{\mathrm{c}}$ for which value $\lambda_{\mathrm{c}}>0$, and there is no solution in the large $|\lambda|$ limit. As a consequence, we found solutions only for aiding flow, $\lambda>0$, with a critical value at $\lambda_{\mathrm{c}} \simeq 0.3119$ limiting the range of solutions. Both solution branches emerging from the saddle-node bifurcation became singular as $\lambda \rightarrow 0$ from above.

Lastly, we examined how the solution behaved with the exponent $m$ for a fixed value of $\lambda$ for both aiding flow, Fig. 14, and opposing flow, Fig. 15. In both cases, we found a solution for all $m>0$ approaching the corresponding asymptotic limit as $m \rightarrow \infty$. For aiding flow, there was a solution for only a limited range of $m<0$. The same applied for opposing flow although now this solution did extend slightly into $m>0$ with one solution branch becoming singular as $m \rightarrow m_{\mathrm{i}}$.

Finally, it is worth thinking about the temporal stability of the similarity solutions. To address this aspect, we can set up an initial-value problem by adding time-dependent terms to the equations with the similarity solutions then being possible large time behaviour of this system. By making a linear perturbation about the similarity solutions proportional to $\mathrm{e}^{\omega t}$ leads to an eigenvalue problem for $\omega$ with its sign determining the stability. Previous studies, though limited, of this and related problems suggest that it is the upper branch solutions, as seen in Fig. 2a, which are stable, the saddle-node bifurcation at $\lambda_{\mathrm{c}}$ leading to a change in the temporal stability with the lower branch being unstable. From this, we might conjecture that the solution branch that proceeds to large $\lambda$ is the stable one, changing stability at a saddle-node bifurcation. What happens when the solution proceeds to large negative $\lambda$ or when there are more than two solutions, as in Figs. 10 and 11, is far from clear and needs further treatment, although this is beyond the scope of the present work.

Acknowledgments The author wishes to thank the referees for their helpful and constructive comments.

Open Access This article is distributed under the terms of the Creative Commons Attribution 4.0 International License (http:// creativecommons.org/licenses/by/4.0/), which permits unrestricted use, distribution, and reproduction in any medium, provided you give appropriate credit to the original author(s) and the source, provide a link to the Creative Commons license, and indicate if changes were made. 


\section{References}

1. Falkner VM, Skan SW (1931) Solutions of the boundary layer equations. Philos Mag 12:132-135

2. Hartree DR (1937) On an equation occurring in the Falkner and Skan's approximate treatment of the equations of the boundary layer. Proc Camb Philos Soc 33:223-239

3. Rosenhead L (ed) (1963) Laminar boundary layers. Clarendon Press, Oxford

4. Stewartson K (1954) Further solutions of the Falkner-Skan equation. Proc Camb Philos Soc 50:454-465

5. Brown SN, Stewartson K (1966) On the reversed flow solutions of the Falkner-Skan equation. Mathematika 13:1-6

6. Craven AH, Peletier LA (1972) Reverse flow solutions of the Falkner-Skan equation for $\lambda>1$. Mathematika 19:135-138

7. Oskam B, Veldman AEP (1982) Branching of the Falkner-Skan solutions for $\lambda<0$. J Eng Math 6:295-308

8. Libby PA, Lui T (1972) Further solutions of the Falkner-Skan equation. AIAA J 22:329-334

9. Fang T, Yao S, Zhang J, Zhong Y, Tao H (2012) Momentum and heat transfer of the Falkner-Skan flow with algbraic decay: an analytic solution. Commun Nonlinear Sci Numer Simul 17:2476-2488

10. Crane L (1970) Flow past a stretching plate. Z Angew Math Phys 21:645-647

11. Banks WHH (1983) Similarity solutions of the boundary-layer equations for a stretching wall. Journal de Mécanique théorique et appliquée 2:375-392

12. Magyari E, Keller B (2000) Exact solutions for self-similar boundary-layer flows induced by permeable stretching walls. Eur J Mech B 19:109-122

13. Weidman PD, Kubitschek DG, Davis AMJ (2006) The effect of transpiration on self-similar boundary layer flow over moving surfaces. Int J Eng Sci 44:730-737

14. Fang T, Yao S, Zhang J, Aziz A (2010) Viscous flow over a shrinking sheet with a second order slip flow model. Commun Nonlinear Sci Numer Simul 15:1831-1842

15. Ishak A, Jafar K, Nazar R, Pop I (2009) MHD stagnation point flow towards a stretching sheet. Physica A 388:3377-3383

16. Wang CY (2011) Review of similarity stretching exact solutions of the Navier-Stokes equations. Eur J Mech B 30:475-479

17. Al-Housseiny TT, Stone HA (2012) On boundary-layer flows induced by the motion of stretching surfaces. J Fluid Mech 706: 597-606

18. Magyari E (2009) Falkner-Skan flows past moving surfaces: an exactly solvable case. Acta Mech 203(1-2):13-21

19. Klemp JB, Acrivos A (1976) A moving-wall boundary layer with reversed flow. J Fluid Mech 76:363-381

20. Merrill K, Beauchesne M, Previte J, Paullet J, Weidman P (2006) Final steady flow near a stagnation point on a vertical surface in a porous medium. Int J Heat Mass Transf 49:4681-4686

21. Hussaini MY, Lakin WD, Nachman A (1987) On similarity solutions of a boundary layer problem with an upstream moving wall. SIAM J Appl Math 47:699-708

22. Riley N, Weidman PD (1989) Multiple solutions of the Falkner-Skan equation for flow past a stretching boundary. SIAM J Appl Math 49:1350-1358

23. Wilks G (1974) A separated flow in mixed convection. J Fluid Mech 62:359-368

24. Wilks G, Bramley JS (1981) Dual solutions in mixed convection. Proc R Soc Edinb 87A:349-358

25. Merkin JH, Mahmood T (1989) Mixed convection boundary layer similarity solutions: prescribed wall heat flux. J Appl Math Phys (ZAMP) 20:51-68

26. Merkin JH (2014) The effects of an outer flow on the unsteady free convection boundary layer near a stagnation point in a heat generating porous medium. Quart J Mech Appl Math 67:419-444

27. Gebhart B, Jaluria Y, Mahajan RL, Sammakia B (1988) Buoyancy-induced flows and transport. Hemisphere Publishing Corp, New York

28. Numerical Algorithm Group (NAG). www.nag.co.uk

29. Merkin JH (1985) On dual solutions occurring in mixed convection in a porous medium. J Eng Math 20:171-179

30. Isak A, Merkin JH, Nazar R, Pop I (2008) Mixed convection boundary layer flow over a permeable vertical surface with prescribed wall heat flux. Z Angew Math Phys 59:100-123

31. Merkin JH, Pop I (2010) Natural convection boundary-layer flow in a porous medium with temperature dependent boundary conditions. Transp Porous Media 85:397-414

32. Lok YY, Merkin JH, Pop I (2012) Mixed convection flow near the axisymmetric stagnation point on a stretching cylinder. Int $\mathbf{J}$ Thermal Sci 59:186-194

33. Roşca N, Roşca AV, Pop I, Merkin JH (2015) Mixed convection boundary-layer flow near the lower stagnation point of a horizontal circular cylinder with a second-order wall velocity condition and a constant surface heat flux. IMA J Appl Math 80:431-451 